\title{
Patterns in the composition of ground-dwelling araneomorph spider communities in the Western Australian wheatbelt
}

\author{
M. S. Harvey', J. M. Waldock', N. A. Guthrie'2, B. J. Durrant ${ }^{2}$ and N. L. McKenzie \\ Department of Terrestrial Invertebrates, Western Australian Museum, Francis Street, \\ Perth, Western Australia 6000, Australia \\ Department of Conservation and Land Management, PO Box 51 Wanneroo, \\ Western Australia 6065, Australia
}

\begin{abstract}
Ground-dwelling araneomorph spiders were sampled at 304 quadrats chosen to represent the geographical extent and diversity of uncleared terrestrial environments in a $205000 \mathrm{~km}^{2}$ area known as the Western Australian wheatbelt. A total of 744 species comprising 39 families were recorded, of which the families Salticidae (121 species), Zodariidae (117), Therididae (80), Lycosidae (61), Oonopidae (46) and Lamponidae (41) exhibited a marked species-level radiation. For analysis, families with a high proportion of arboreal species, and quadrats that were often flooded or overtly affected by secondary salinity, were excluded. Thus, a total of 622 species from 240 quadrats were analysed, with an average of $21.9(\mathrm{s.d} .=8.3)$ species per quadrat. Most of the variation observed in the patterns of species composition could be explained in terms of summer temperature, precipitation seasonality, soil salinity and $\mathrm{pH}$ attributes. Assemblage species richness was constrained by soil salinity, except for the Lycosidae which showed a positive relationship.
\end{abstract}

\section{INTRODUCTION}

South-western Australia represents a region that is internationally recognized for its exceptional diversity of terrestrial life, according it the status of one of the world's top-20 biodiversity hotspots (Myers of al., 2000). This ranking was largely based upon the remarkable radiations of the vascular flora, but it has become increasingly obvious that many components of the terrestrial invertebrate fauna are very species-rich and that high proportions of the fauna are endemic to the region (Hopper of al., 1996). Some terrestrial and aquatic invertebrates are also postulated as possessing high numbers of short-range endemic taxa (Harvey, 2002). We confined our study to araneomorph spiders which are easy to collect in pit-fall traps and for which we have sufficient expertise to undertake species-level identifications.

Although sporadic descriptions of Western Australian spiders were made by $19^{\text {th }}$ century Furopean taxonomists on specimens collected from King George Sound and the Swan River Colony, the first comprehensive publication was by Eugene Simon (1908, 1909) who published on the collections of a German Expedition led by $W$. Michatsen and R. Hartmever to south-western Australia in 1905. A great variety of species were named, but unfortunately few of the names are currently in use due to the poor descriptions and paucity of illustrations.
Very few araneomorph spider families are currently well known in southern Western Australia. Taxonomic revisions have been published for only a few taxa, including: Ammoxenidae (Platnick, 2002), Anapidae (Platnick and Forster, 1989), Filistatidae (Gray, 1994), Gallieniellidae (Platnick, 2002), Hersilidae (Baehr and Baehr, 1987, 1989, 1992, 1993, 1995, 1998), Lamponidae (Platnick, 2000), Nicodamidae (Harvey, 1995), Orsolobidae (Forster and Platnick, 1985), some Salticidae (e.g. Zabka, 1990, 1991, 1992a, 1992b, 2000; Zabka, 2001), some Sparassidae (Davies, 1994; Hirst, 1989, 1991a,b, 1992), Trochanteridae (Platnick, 2002) and most Zodariidae (Bathr and Jocqué, 2000, 2001; Jocque and Baehr, 2001; Jocqué and Baehr, 1992; Bathr and Churchill, 2003; Baehr, in press)

Over the past 15 years, several broad-scale biotic surveys have been conducted in Western Australia, and spiders were one of the groups targeted for detailed analysis to discern how the spider fauna was distributed across the landscape. The first was an examination of the fauna of the Kimberley rainforests in which spiders were examined in detail by Main (1991). A rich assemblage of both cursorial and web-building types was revealed.

Perhaps the most comprehensive study was in the southern Carnarvon Basin where a pitfall trapping program revealed a total fauna of at least 500 species (Harvey et al., 2000); Main if al., 2000). 
Unique patterns of endemism were found, and spatial patterns in the composition of the spider fauna were strongly correlated with climatic and soil attributes.

The dataset we investigated in this paper was collated as part of a biodiversity survey of the Western Australian wheatbelt (Figure 1), an inland area of some $205000 \mathrm{~km}^{2}$ ranging from Geraldton to Esperance, to discern patterns of endemism in a highly fragmented and disturbed landscape. We provide the first account of the Western Australian wheatbelt's fauna of ground-dwelling araneomorph spiders, and explore patterns in community composition in terms of measurable attributes of the area's physical environment, including salinity.

\section{METHODS}

\section{Study area}

The wheatbelt study area has an area of 205000 $\mathrm{km}^{2}$ and comprises all or part of five biogeographical regions (Figure 1). The inland boundary is a little to the west of the $300 \mathrm{~mm}$ isohyet. The western boundary is east of the 600 $\mathrm{mm}$ isohyet. Along the southern coast, the southern boundary approximates the $550 \mathrm{~mm}$ isohyet but avoided near-coastal environments. Its semi-arid to sub-humid climate is influenced by temperate weather systems (mainly winter rainfall, see Bureau of Meteorology, 2001), and its surface hydrology is characterised by low gradients, high potential salt

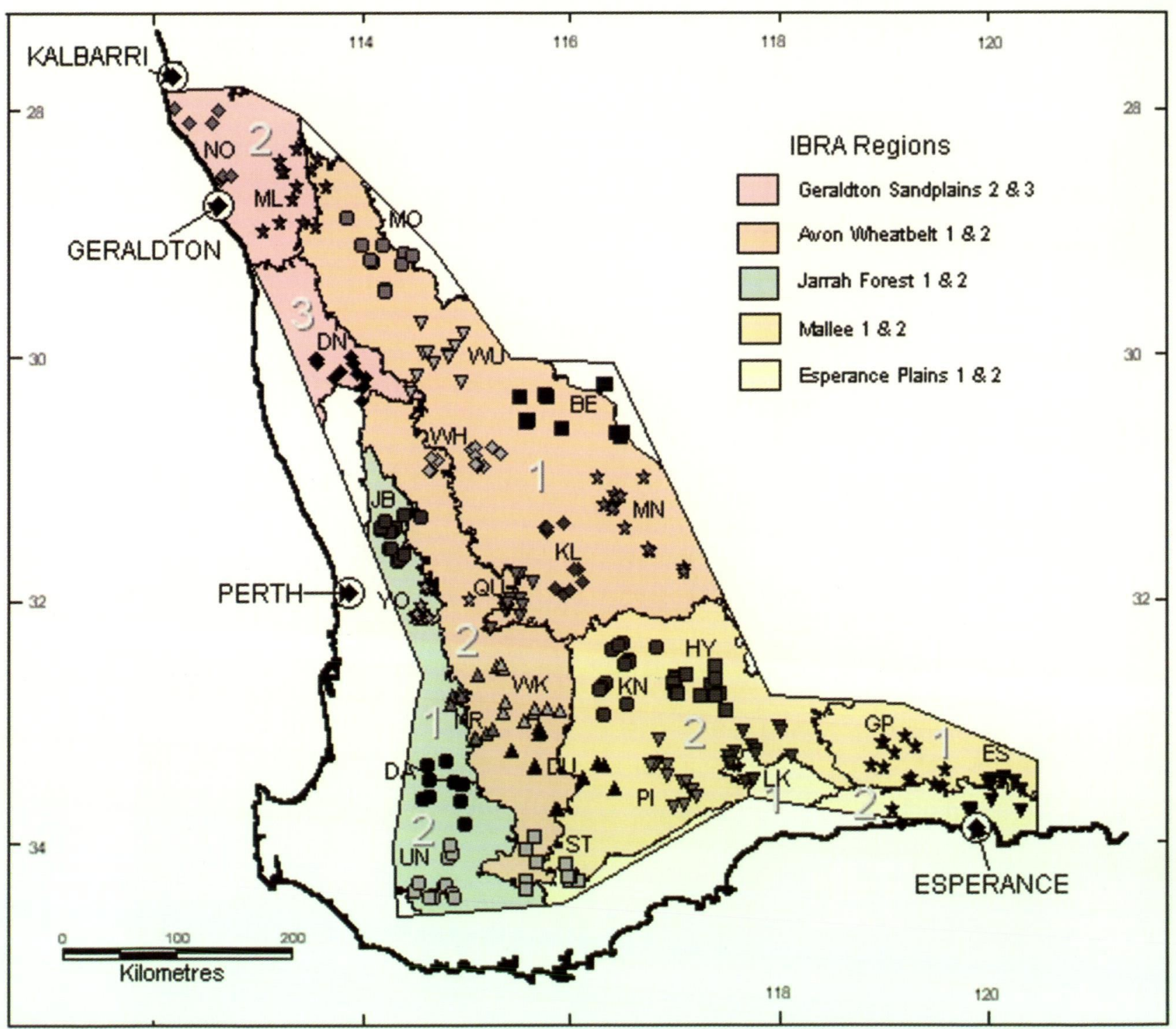

Figure 1 Wheatbelt study area, showing the 24 survey areas and the positions of the 12 to 13 individual quadrats in each survey area. Precise quadrat co-ordinates are provided in Appendix 1. The coloured areas indicate bioregional boundaries (Environment Australia, 2000; modified from Beard, 1980). The fine lines internal to the bioregions are sub-regional boundaries defined from IBRA version 5 (Environment Australia, 2000; Morgan, 2001). 
loads and high variability in flows (Commander et al., 2002; George and Coleman, 2002; Hatton and Ruprecht, 2002; Schoknecht, 2002). Overall, 74\% of the study area has been cleared for agriculture; its remaining bushlands are isolated woodland and shrubland mosaics embedded in fields of wheat, lupin and canola. Extensive areas are now affected by dryland salinity (George et al., 1995).

In phytogeographical terms the study area comprises the semi-arid part of the South-Western Botanical Province (Beard, 1980). Detailed descriptions of the vegetation in the study area are provided by Beard (1980, 1981, 1990). The study area's substrates are detailed by Chin (1986), Mulcahy and Hingston (1961) and Myers and Hocking (1998). Most of it is an undulating plateau of Tertiary duricrust overlying Archaean granitic and metamorphic rock strata of the Yilgarn Craton. The plateau is variously eroded into elluvial and colluvial spillway sand deposits supporting a variety of shrublands, and dissected to expose valley-slope and -floor units characterised by duplex soils derived from the Archaean basement strata and supporting woodlands. Except in the south-west, chains of salt-lakes also occur along these valley floors. Although the north-western (Geraldton Sandplains) and south-eastern (Esperance Plains) parts of the study area are on different geological basements (e.g. Morgan and Peers, 1973), their geomorphic units could be classified into the same landform catena according to soil profile attributes.

\section{Field sampling strategy}

Sampling strategy is discussed in McKenzie et al. (2004). The study area was divided into 24 survey areas of similar size so that sampling was evenly dispersed across the study area's areal extent (Figure 1). Ten to 11 quadrats each of one hectare were positioned to sample the geomorphic profile of each of 24 survey areas (Figure 1). In each survey area, a quadrat was positioned in a typical example (in terms of vegetation and soil) of each of the main geomorphological units (Baxter and Lipple, 1985; Chin, 1986; Morgan and Peers, 1973; Mulcahy and Hingston, 1961). An additional one or two quadrats were positioned to sample examples of these units that were affected by groundwater salinity. The quadrats chosen were the least disturbed examples we could find. Even the sites selected to represent

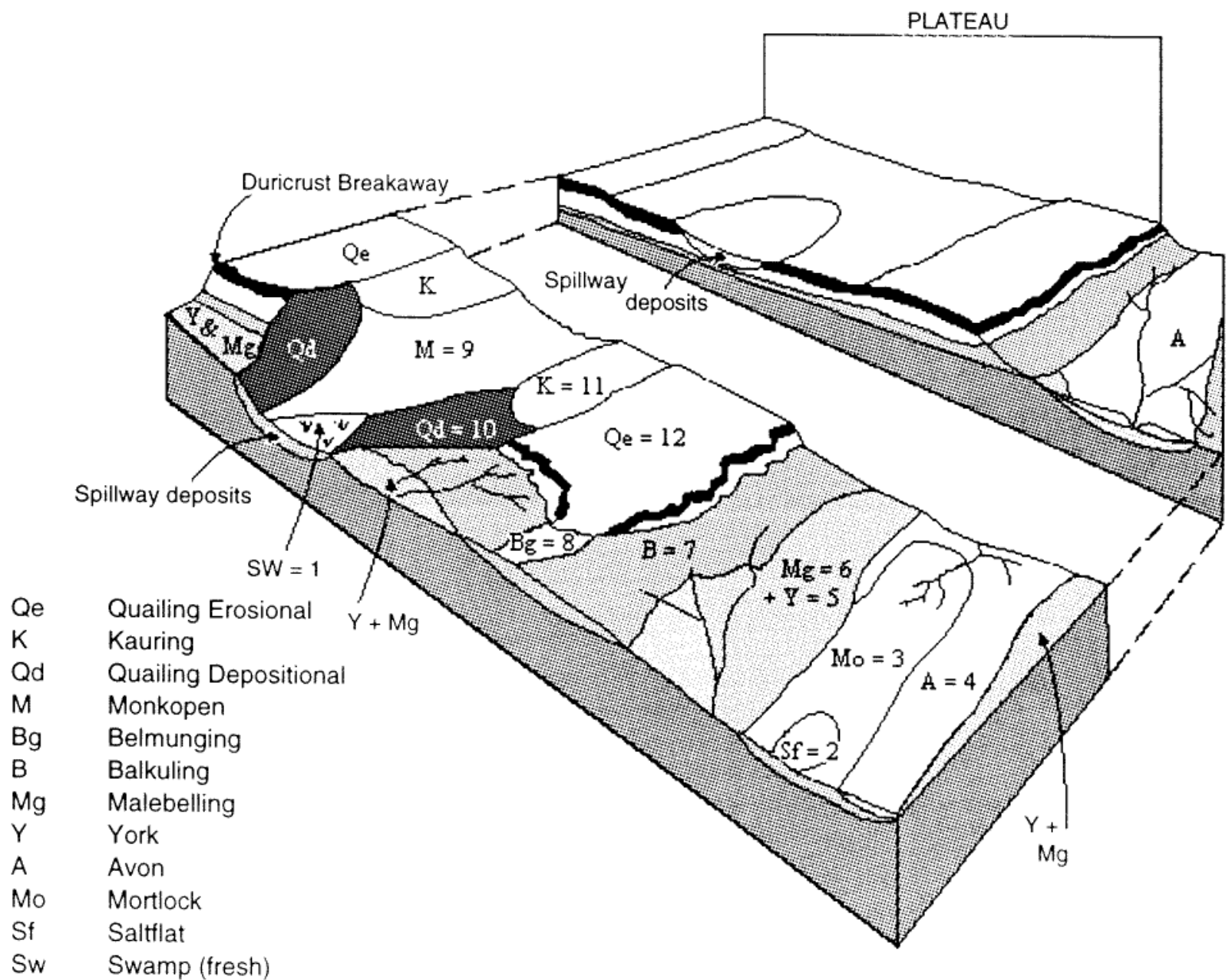

Figure 2 Wheatbelt landforms, modified from Mulcahy and Hingston (1961). The landscape's plateau profile comprises the duricrusted Tertiary laterite plateau and its derived spillway sands, while the dissection profile comprises finer textured soils derived from bedrock and pallid-zone clays beneath the duricrust. 
secondarily salt-affected sites were positioned to minimise proximity to wheatfields and avoid evidence of other disturbances such as rubbish disposal, gravel extraction and previous clearing. Locations of the 304 quadrats are provided in Appendix 1.

A consistent landform model was used to position the individual quadrats (Figure 2), so an equivalent cross-section of the landscape profile's main components was sampled in each survey area. This nested, stratified sampling allowed an analytical option in which each survey area was treated as a single landscape-scale relevé; by pooling the lists of species from a survey area's 12 or 13 quadrats, we might suppress the influence of local substrate on the classification structure, thereby exposing broad-scale patterns for analysis.

The spiders on each quadrat were sampled using five 2-litre, glycol pits left open for one calender year (1825 pit trap nights per quadrat). Pits were 25 $\mathrm{cm}$ deep, $12 \mathrm{~cm}$ in diameter, contained 0.4 litres of glycol-formalin $(320 \mathrm{ml}$ ethylene glycol, $64 \mathrm{ml}$ tap water, $16 \mathrm{ml}$ formaldehyde) and were set flush with the ground surface. Each pit was protected by a square plate of wood $(0.15 \times 0.15 \mathrm{~m})$ suspended 5 $\mathrm{cm}$ above the pit's mouth. The five pits on each quadrat were positioned in different microhabitats - bare ground, leaf litter patches under trees, beside rotting logs, under low shrubs etc. They were always at least five metres apart.

Quadrats in the YO, QU, KL, MN, NR, WK, KN and $\mathrm{HY}$ survey areas were sampled from October 1997 to September 1998. NO, ML, MO, WU, JB, WH and BE were sampled from September 1998 to October 1999, and DA, DU, PI, LK, UN, ST, GP and ES from October 1999 to October 2000. Every four to six months, specimens were removed from the pits and fluids replenished. After washing, sorting and identification, the specimens were lodged in the collection of the Western Australian Museum.

\section{Physical attributes at quadrats}

Landform units in survey areas on the Yilgarn Craton were numbered from 1 to 12 according to their position in the landscape profile (see Figure 2, McKenzie et al. 2004). Units belonging to the dissection profile were numbered from 1 to 7 , while spillway sand and duricrust units associated with the old plateau profile were numbered from 9 to 12 . Unit $\mathrm{Bg}$ in which spillway sand mantles a clay belonging to the dissected valley profile was assigned to number 8 . Units at the bottom of the dissection profile included fresh water swamps (unit 1) and saline flats (unit 2). The highest unit in the landscape was duricrust pavement of the old Tertiary plateau (unit 12). Quadrats in survey areas on geological basements other than the Yilgarn Craton (those in the Esperance Plains and Geraldton Sandplains bioregions) were positioned using the relevant 1:250 000 maps of surface lithology (e.g. Morgan and Peers, 1973), and arbitrarily assigned to our 12-class landform catena according to their soil profile (texture and horizon sequence), position in the relevant landscape's profile and soil origin. Thus, a deep, low-level sandsheet wouid be assigned to the same number as the lowest of the spillway sand units on the Craton (Monkopen $=9$, in Figure 2). This approximation was considered to be acceptable because data taken from the quadrats themselves were used in subsequent analyses.

Eighteen climatic attributes were derived for each quadrat using ANUCLIM (McMahon et al., 1995). These comprised annual and seasonal average and range values for temperature and precipitation (Appendix 2). Soil attributes were also recorded from each quadrat (Appendix 3), including nitrogen, phosphorus, potassium, $\mathrm{pH}$, electrical conductivity, organic carbon, clay-silt-sand percentages and magnesium. Eight landform and five vegetation attributes were also generated (Appendix 1), including latitude, longitude, elevation, landform unit, soil drainage category, slope, salinity risk, salinity class, litter/log cover, tree cover, shrub cover, herb cover and habitat complexity (modified from Newsome and Catling, 1979). Latitude and longitude values were determined using a hand-held GPS accurate to \pm 30 $\mathrm{m}$. Explanation of the salinity attributes is provided in McKenzie et al. (2004) and in Appendix 1. Because landform had already been used to position the quadrats in each survey area, it could not also be used as an attribute in the compositional analysis.

\section{Analytical strategy}

The analytical approach taken in this paper was based on the assumption that spatial distribution reflects an underlying correlation with environmental factors (Austin, 1991; Clarke, 1993). It is an exploratory design, and interpretation is based on deductive rather than inductive logic (Oksanen, 2001). No experimental design has been implemented to assess a null hypothesis (Austin and McKenzie, 1988), so alternative hypotheses are not excluded. Inferential statistics are used to test patterns observed in the empirical data.

The relative responses of wheatbelt araneomorph species to our pitfall traps are unknown as are the year-to-year dynamics of population numbers in these communities. We also recognise other limitations in sampling techniques which were aggravated by staff and time limitations. Because these issues precluded reliable estimates of species abundance, we chose to restrict our analyses to data on the presence-absence of taxa at individual quadrats (Austin, 1984; McKenzie et al., 1991). Thus, the input data utilised for the compositional analyses were survey area- $x$-genus, survey area- $x$ - 
species and quadrat-x-species matrices. Species recorded at only a single quadrat (singletons) were excluded from compositional analysis because they convey little information on patterns.

To assess whether records of a species were more geographically localised than would be expected by chance, the average distance between the quadrats at which a species was recorded was calculated and compared with 1000 trials using an equivalent number of randomly arrayed records (quadrat intersections). The probability $(P)$ of achieving the observed average distance was then assessed from the distribution of the 1000 randomly derived values.

To gain a clear separation between species of nonsaline environments and those tolerant or adapted to saline conditions, the 51 quadrats affected by secondary salinity were excluded from the quadrat$x$-species matrix (see McKenzie et al., 2003). This separation provided a basis for interpreting the species composition of assemblages on the saline quadrats in the absence of extrinsic data on the habitat preferences of virtually any of the araneomorphs in the samples. Thirteen additional quadrats were eliminated from this analysis because they were flooded for long periods during the trapping program (10 in ephemeral fresh water swamps, as well as PI04, UN13 and WK02). This left 240 quadrats in the quadrat-x-species matrix, but did not distort the stratification (see Table 1 in McKenzie et al., 2003).

Cluster analysis (from PATN, Belbin, 1995) was used to expose patterns of species composition in the data matrices. The Czekanowski measure (Czekanowski, 1932) was used to compare the quadrats according to their species similarities, because it is known to provide a robust measure of ecological distance (Faith et al., 1987). A modified version of the unweighted pair group arithmetic averaging (UPGMA - Belbin, 1995; Sneath and Sokal, 1973) hierarchal clustering strategy was used, with the clustering parameter (Beta) set to -0.1. The partition structure of the resulting dendrogram was used as a summary of compositional patterns in araneomorph genera/species across the study area. Environmental attributes that conformed to the partition structures were assessed for statistical significance using Kruskall-Wallis one way analysis of variance by ranks (in the computer package STATISTICA, Statsoft, 2001). The negative exponential smoothing function in STATISTICA was used to interpolate climatic surfaces across the study area thereby illustrating geographic patterns in climatic attributes that were found to be significant.

\section{RESULTS}

The infraorder Araneomorphae was represented in the survey by 39 families, and a total of 24640 specimens were identified to species level. The numbers of specimens identified from each quadrat were not particularly high despite large sampling effort (average 81.1, s.d. 49.6, range 2 - 316), and there are substantial differences between quadrats that may indicate differences in sampling effectiveness and lead to bias in comparisons of quadrat richness. Some of the poorest quadrats were those on salt-flats or in low-lying salt-affected areas. An additional six months of sampling at a sub-set of the salt-flat quadrats revealed no additional araneomorph species (Durrant and Guthrie, 2004), so we proceeded with analyses of richness on the basis that our samples adequately reflect trends. This sampling issue is investigated further under the heading Richness, below.

The representatives of five families were not identified to species-level due to time and resource constraints, as well as uncertainty regarding the unresolved taxonomic boundaries at the species level. These five families (Ctenidae, Gnaphosidae, Miturgidae, Sparassidae and Zoridae) are not listed below and were excluded from the analyses.

Observations on species geographical occurrence that are included in the annotated list below are derived from Table 1.

\section{Agelenidae}

Two species assigned to the Agelenidae were recorded in the survey. The first, Genus $1 \mathrm{sp}$. 1, was found at the KL survey area, whilst the second, Genus 1 sp. 2, was found at UN and WK.

\section{Amaurobiidae}

The family Amaurobiidae was represented in the survey by a diverse assemblage of 27 species. Four species were found at more than 20 quadrats (Genus 2 sp. 01, Genus 3 sp. 01, Genus 3 sp. 02 and Genus 3 sp. 16) and seven species were found at only a single quadrat. The amaurobiid fauna of Western Australia is poorly known, and the systematic position of many of the species here placed in this family may reside in other families.

\section{Amphinectidae}

The survey revealed 11 species attributed to the Amphinectidae, most of which were found in just a few quadrats. They were predominately recorded from southern study sites.

\section{Anapidae}

The anapid fauna of southern Western Australia is represented by the sole genus Chasmocephalon, a genus that is also found in eastern Australia along with a variety of other genera (Platnick and Forster, 1989). All anapids are tiny spiders less than $2-3 \mathrm{~mm}$ in length. Three species of Chasmocephalon were 
Table 1 List of araneomorph taxa identified during the survey; the families Ctenidae, Gnaphosidae, Miturgidae, Sparassidae and Zoridae were omitted from the analysis due to time constraints. The study sites (see Figure 1) are arranged approximately from north-west to south-east and each cell records the number of quadrats from which each species was recorded.

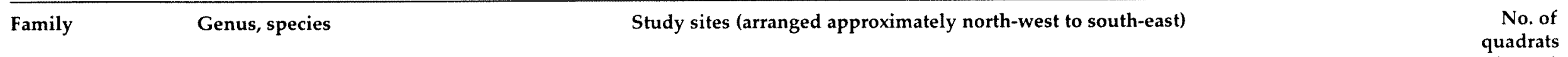

NO ML DN MO WU WH BE JB YO QU KL MN NR WK KN HY DA UN DU ST PI LK GP ES (n=304)

Agelenidae

Agelenidae

Amaurobiidae

Amaurobiidae

Amaurobiidae

Amaurobiidae

Amaurobiidae

Amaurobiidae

Amaurobiidae

Amaurobiidae

Amaurobiidae

Amaurobiidae

Amaurobiidae

Amaurobiidae

Amaurobiida

Amaurobiidae

Amaurobiidae

Amaurobiidae

Amaurobiidae

Amaurobiidae

Amaurobiidae

Amaurobiidae

Amaurobiidae

Amaurobiidae

Amaurobiidae

Amaurobiidae

Amaurobiida

Amaurobiidae

Amaurobiidae

Amphinectidae

Amphinectidae

Amphinectidae

Amphinectidae

Amphinectidae

Amphinectidae

Amphinectidae

Amphinectidae

Amphinectidae
Genus $1 \mathrm{sp.} 01$

Genus 1 sp. 02

Genus $1 \mathrm{sp} .01$

Genus 2 sp. 01

Genus 2 sp. 02

Genus 2 sp. 03

Genus $3 \mathrm{sp} .01$

Genus $3 \mathrm{sp} .02$

Genus 3 sp. 03

Genus 3 sp. 04

Genus 3 sp. 05

Genus $3 \mathrm{sp} .06$

Genus $3 \mathrm{sp} .07$

Genus 3 sp. 08

Genus 3 sp. 09

Genus 3 sp. 10

Genus 3 sp. 11

Genus 3 sp. 12

Genus 3 sp. 13

Genus 3 sp. 14

Genus 3 sp. 16

Genus 4 sp. 01

Genus 4 sp. 02

Genus 4 sp. 03

Genus 4 sp. 04

Genus 5 sp. 01

Genus 7 sp. 01

Genus 8 sp. 01

Genus 9 sp. 01

sp. 01

sp. 02

sp. 03

sp. 04

sp. 05

sp. 06

sp. 07

sp. 08

sp. 10

\begin{tabular}{|c|c|c|c|c|c|c|}
\hline & 1 & 1 & & & & \\
\hline 2 & 3 & & 1 & 3 & 2 & 3 \\
\hline & & 1 & & & & \\
\hline 1 & & 4 & & & 2 & \\
\hline
\end{tabular}

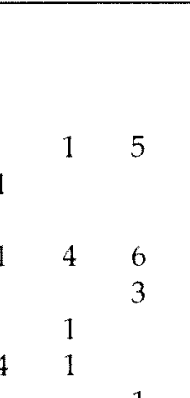

1

1

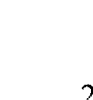

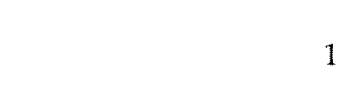

$\begin{array}{llll}1 & & 1 & 2 \\ 1 & 2 & 1 & \end{array}$

225

5

2

4

2

11

1

2

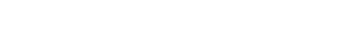

更

1
1


Anapidae

Chasmocephalon sp. 01

Anapidae

Chasmocephaton sp. 01
Chasmocephalon sp. 02

Anapidae

Chasmocephalon sp. 03

Araneidae

sp. 03

Araneidae

sp. 04

Araneidae

Araneidae

sp. 05

Araneidae

sp. 06

sp. 07

Araneidae

sp.08

Arancidae

Araneidae

Araneidae

Araneidae

Araneidae

Araneidae

Araneidae

Araneidae

Araneidae

Araneidae

Araneidae

Araneidae

Araneidae

Araneidae

Araneidae

Araneidae

Cyatholipidae

Cyatholipidae

Cycloctenidae

Cycloctenidae

p. 10

sp. 11

sp. 12

sp. 13

sp. 14

p. 16

Argiope protensa

Argiope trifascinta

Austracantha minax

Dolophones sp. 01

Dolophones sp. 02

Dolophones sp. 03

Eriophora biapicata

Eriophora transmarina

Eriophora sp. 01

Eriophora sp. 02

Genus $X$ sp.

Genus $Y$ sp.

Genus Z sp.

Matilda sp. 01

Matilda sp. 02

Genus 1 sp. 01

Deinopidae

Genus $1 \mathrm{sp} .02$

Desidae

Deinopis sp.

sp. 01
sp. 02

sp. 02

Desidae sp.03

Desidae sp. 04

Desidae $\quad$ sp. 05

Desidae sp. 06

Desidae sp. 07

Desidae sp. 08

Desidae sp. 10

Desidae

sp. 11 


Family Genus, species Study sites (arranged approximately north-west to south-east) No. of

NO ML DN MO WU WH BE JB YO QU KL MN NR WK KN HY DA UN DU ST PI LK GP ES (n=304)

\begin{tabular}{|c|c|c|c|c|c|c|c|c|c|c|c|c|c|c|c|c|c|c|c|c|c|c|c|c|c|}
\hline Desidae & Genus $1 \mathrm{sp} .01$ & & & & & 2 & & 1 & & 1 & & 1 & & & 1 & 1 & & & 1 & 1 & 1 & 3 & 2 & & 15 \\
\hline Desidae & Genus $1 \mathrm{sp} .02$ & & & 2 & & & 1 & 1 & & 1 & & & & & & & & & 1 & & & & & & 6 \\
\hline Desidae & Genus 1 sp. 03 & & 1 & & 1 & 2 & 1 & 2 & & & & & 1 & & & & 1 & & 2 & & 1 & 1 & & & 13 \\
\hline Desidae & Genus 1 sp. 04 & & & & & 2 & 1 & 2 & 1 & & & 1 & & & & & & & & & & & & & 7 \\
\hline Desidae & Genus 1 sp. 05 & & & & 1 & & & & 1 & 1 & 1 & & 1 & 2 & & & & & 2 & & & & & & 9 \\
\hline Desidae & Genus 1 sp. 06 & & & & & & & & & & & & & & & & 1 & & & & & & 2 & 3 & 6 \\
\hline Desidae & Genus 1 sp. 07 & & & & & & & & & & & & & & & & & & & 1 & & & & & 1 \\
\hline Desidae & Genus 1 sp. 08 & & & & & & & 1 & & 1 & & 1 & & & 1 & 1 & & & & & 1 & & & & 6 \\
\hline Desidae & Genus 1 sp. 09 & & & & & & & & & & & & & & & & & & 1 & & & & & & 1 \\
\hline Desidae & Genus 1 sp. 10 & & 1 & & & 1 & & & & & & & & & & & & & & & & & 1 & & 3 \\
\hline Desidae & Genus 1 sp. 11 & & & & & & & & & & & & & 1 & & & & & & & & 1 & & & 2 \\
\hline Desidae & Genus 1 sp. 12 & & & & & & & & & & 2 & & & & & & & & & & & & & & 2 \\
\hline Desidae & Genus $2 \mathrm{sp} .01$ & 1 & 1 & 3 & & 2 & & & 1 & & & 2 & & & 3 & & & 1 & 1 & & & & 1 & & 16 \\
\hline Desidae & Genus 2 sp. 02 & & & & & 2 & & & 1 & & & & & & & & & & & & & & 1 & & 4 \\
\hline Desidae & Genus 3 sp. 01 & & & & & & & & & & & & & 1 & 1 & & & 1 & & & & & & & 3 \\
\hline Desidae & Genus $3 \mathrm{sp} .02$ & & & 1 & 1 & & & 1 & & & 3 & 1 & 2 & & & & 2 & & 2 & & & 2 & & & 15 \\
\hline Desidae & Badumna sp. & 1 & 1 & & 1 & 1 & & 3 & & & & & & & & & & & & & & & & & 7 \\
\hline Desidae & Desidae type?? sp. 01 & & & & 1 & & 1 & & & & 1 & & & & 1 & 2 & & & 1 & 1 & & & & 1 & 9 \\
\hline Desidae & Desidae type?? sp. 02 & 1 & & & & & & & & & & & & & & & & & & & & & & & 1 \\
\hline Desidae & Genus $1 \mathrm{sp} .13$ & & & & & & & & & & & & & & & & & 2 & & & & & & & 2 \\
\hline Desidae & Genus $1 \mathrm{sp} .14$ & & & & & & & & & & & & & & & & & & & & & & 1 & & 1 \\
\hline Desidae & Laestrygones sp. 01 & & & & & & & & & & & & & & & & 2 & & 2 & 1 & 2 & 3 & 2 & 3 & 15 \\
\hline Desidae & Large Olive A & & & & & & & 1 & & & & & & & & & 2 & 4 & & & & & & & 7 \\
\hline Desidae & Large Olive B & & & & & & & & & & & & & & & & & & & & & & & 3 & 3 \\
\hline Desidae & Large Olive C & & & & & & & 1 & 1 & & & & & & & & 1 & 1 & 1 & & & & & & 5 \\
\hline Desidae & Large Olive D & & & & & & & 1 & 2 & & & & & & & & 7 & & 3 & & & & & & 13 \\
\hline Desidae & Large Olive E & & & & & & & 2 & & & & & & & & & 2 & & & 1 & & & & & 5 \\
\hline Desidae & Large Olive F & & & & & & & 1 & 1 & & & & & & & & 1 & 2 & 1 & & & & & & 6 \\
\hline Desidae & Large Olive $\mathrm{G}$ & & & & & & & 1 & & & & & & & & & & & & & & & & & 1 \\
\hline Filistatidae & Wandella barbarella & 3 & 2 & 5 & 1 & 4 & 2 & 1 & 1 & 1 & 1 & & & & 3 & & & & & & & & & & 24 \\
\hline Gallieniellidae & Genus $1 \mathrm{sp} .01$ & & & & & & & & & & & & & & & & 1 & & 1 & & & & & & 2 \\
\hline Gallieniellidae & Meedo houstoni & & & & 2 & & 1 & & & & & & & & & & & & & & & & & & 3 \\
\hline Gallieniellidae & Meedo sp. 01 & & & & & & & & & & & & & & & & & & 1 & & & & & & 1 \\
\hline Gallieniellidae & Meedo sp. 02 & & & & 1 & 1 & & & & & & 1 & & & & & & & & & & & & & 3 \\
\hline Gallieniellidae & Meedo sp. 03 & & & & & & & & & & 1 & 1 & & & & & & & & & & & & & 2 \\
\hline Gallieniellidae & Meedo sp. 05 & & & & & & & & & & & & & & & & 1 & & 2 & & & & & & 3 \\
\hline Hahniidae & sp. 01 & & & & 1 & & & 1 & & 1 & & & & & 1 & 1 & 1 & & & & & & & & 6 \\
\hline Hahniidae & sp. 02 & & & & & & & & & & & & & & 1 & & 1 & & & & & & & & 2 \\
\hline Hahniidae & sp. 03 & & & & & & 1 & & & & & & & & & & & & 1 & & & & & & 2 \\
\hline
\end{tabular}


Lamponidae

Lamponidae

Lamponidae

Lamponidae

Lamponidae

Lamponidae

Lamponidae

Lamponidae

Lamponidae Lamponina elongata

Lamponidae

Lamponidae

Lampona quinqueplas

Lampona sp. 01

Lampona sp. 02

Lampona sp. 03

Lampona sp. 04

Lampona sp. 05

Lampona sp. 06

Lampona sp. 07

Lamponata daviesa

Lamponega forceps

Lamponella ainslie

Lamponina scutato

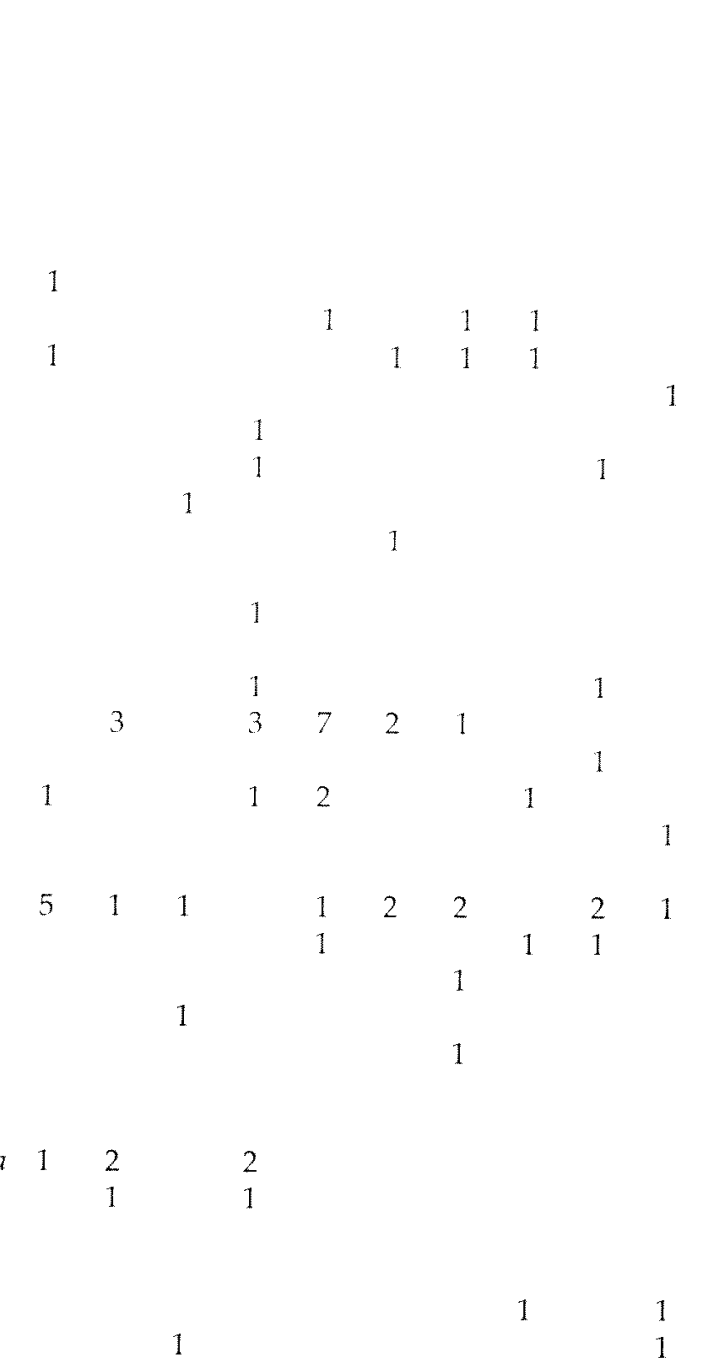

Lamponina sp. 01

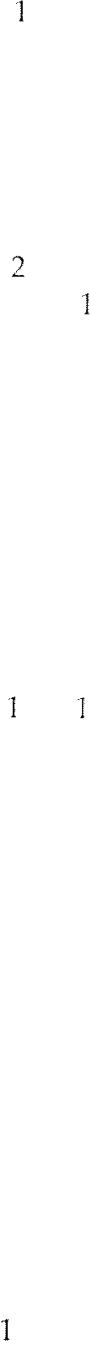

$\begin{array}{ll}3 & 4 \\ & \\ 2 & \\ 2 & \\ & 1\end{array}$


Lamponidae

Lamponidae

Lamponidae

Lamponidae

Lamponidae

Lamponidae

Lamponidae

Lamponidae

Lamponidae

Lamponidae

Lamponidae

Lamponidae

Linyphiidae

Linyphiidae

Linyphiidae

Linyphiidae

Linyphiidae

Linyphiidae

Linyphiidae

Linyphiidae

Linyphiidae

Linyphiidae

Linyphiidae

Linyphiidae

Linyphiidae

Linyphiidae

Linyphiidae

Linyphiidae

Linyphiidae

Linyphiidae

Linyphiidae

Linyphiidae

Linyphiidae

Linyphiidae

Linyphiidae

Linyphiidae

Linyphiidae

Linyphiidae

Linyphiidae
Lamponina sp. 02

Lamponina sp. 03

Lamponusa gleneagle

Longepi woodman

Notsodipus domain

Notsodipus muckera

Notsodipus quobba

Notsodipus sp. 01

Prionosternum porongorup

Prionosternum scutatum

Pseudolampona boree

Queenvic mackay

$$
\text { sp. } 01
$$

sp. 02

sp. 03

sp. 04

sp. 05

sp. 06

sp. 07

sp. 08

sp. 09

sp. 10

sp. 11

sp. 12

sp. 13

sp. 14

sp. 15

sp. 16

sp. 17

sp. 18

sp. 19

sp. 20

sp. 21

sp. 22

sp. 23

sp. 24

sp. 25

sp. 26

sp. 27

\section{C}

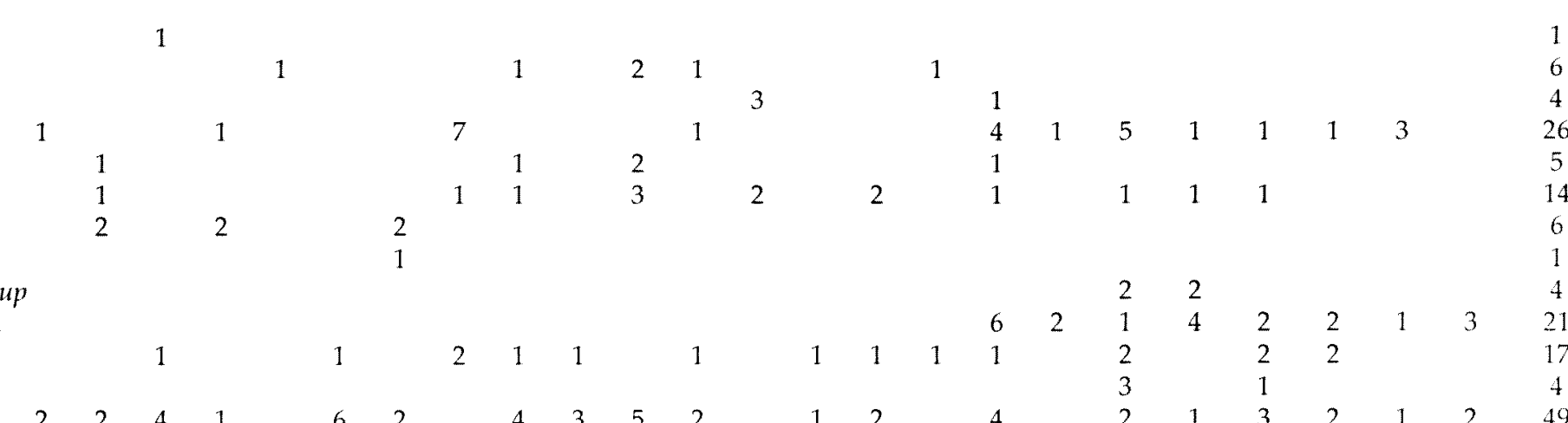

6
4
5
14
6
1
4
17
4

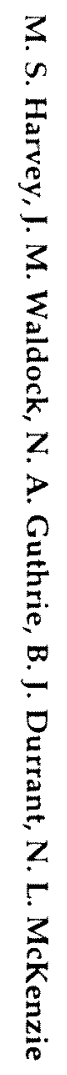


Ostearius melanopygius

sp. 01

Liocranidae

sp. 02

sp. 04

sp. 05

sp. 06

Liocranidae

Liocranidae

sp. 07

Liocranidae

sp. 09

Liocranidae

Lycosidae

sp. 12

Lycosidae

Artoria flavimamus

Artoriasp. 01

Artoria sp. 02

Artoria sp. 03

Lycosidae

Artoria sp. 04

Lycosidae

Artoria sp. 05

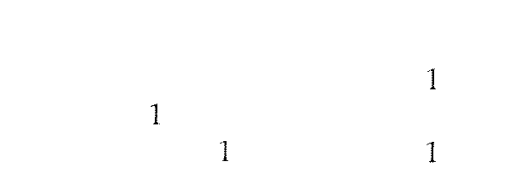

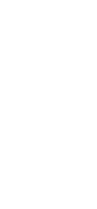

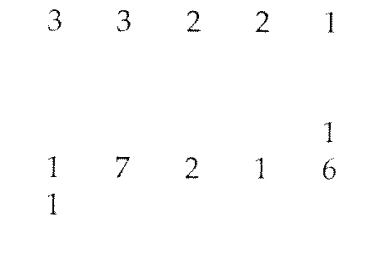

3

32

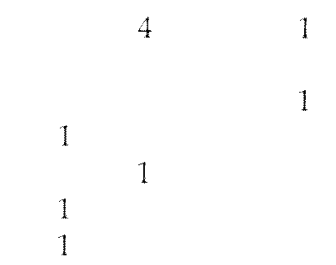

22

$\begin{array}{lll}3 & 1 & 3 \\ & 1 & \\ & 2 & 1\end{array}$

11

$\begin{array}{ll}2 & 1 \\ 2 & 2\end{array}$

1
6
1

1

$\begin{array}{cccc}1 & 1 & & 1 \\ 1 & 2 & 1 & 2 \\ 3 & 2 & 2 & 2 \\ 1 & 3 & & 2 \\ 4 & 10 & & 8 \\ & 1 & 4 & 1 \\ & 1 & 4 & 3 \\ 6 & 1 & 2 & 2\end{array}$

1




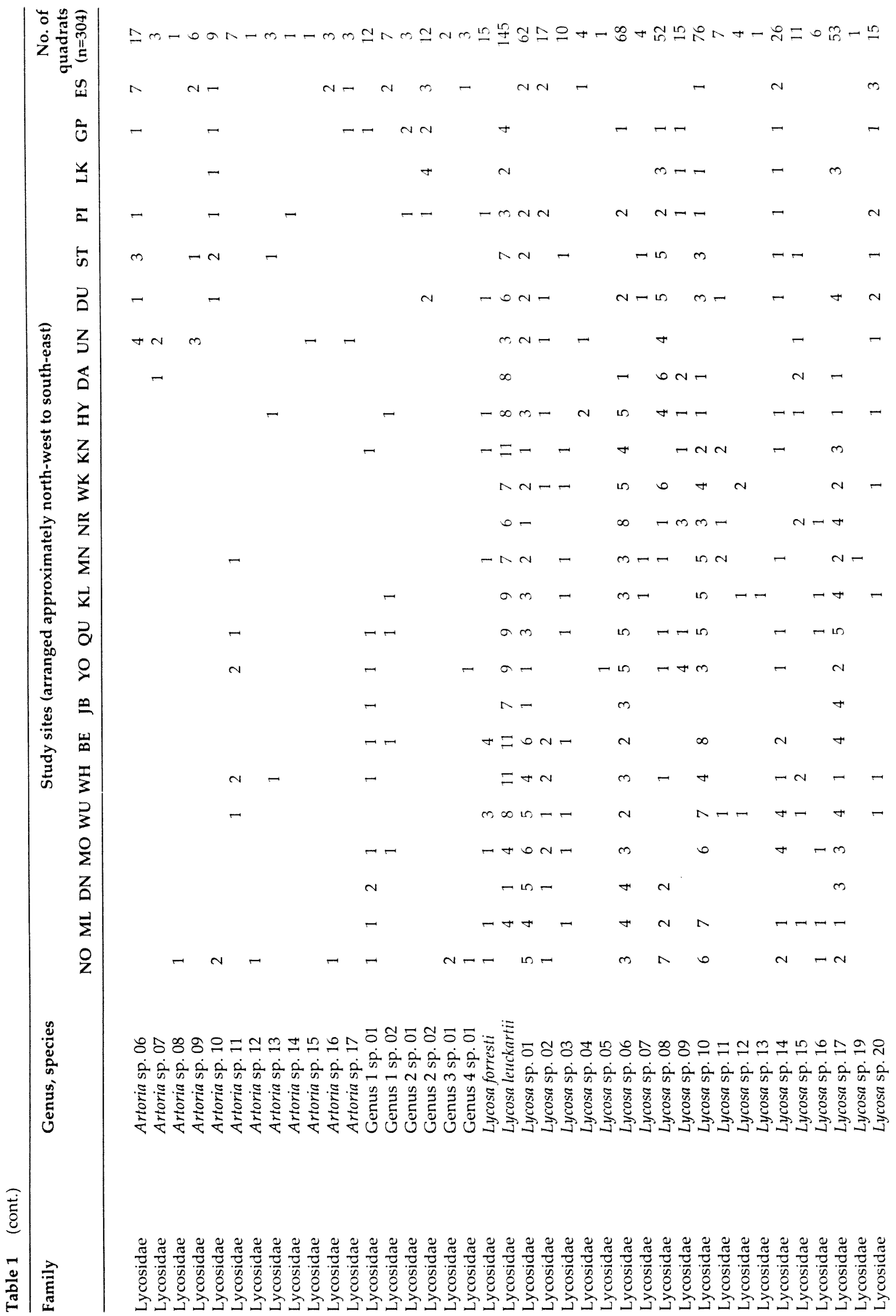


Micropholcommatidae Micropholcomma? sp. 01

Micropholcommatidae Micropholcomma? sp. 02

Micropholcommatidae Micropholcomma? sp. 03

Micropholcommatidae Micropholcomma? sp. 04

Micropholcommatidae Micropholcomma? sp. 06

Micropholcommatidae Micropholcomma? sp. 08

Micropholcommatidae Micropholcomma? sp. 10

Micropholcommatidae Micropholcomma? sp. 11

Micropholcommatidae Micropholcomma? sp. 12

Micropholcommatidae Micropholcomma? sp. 13

Micropholcommatidae Textricella sp. 0

Micropholcommatidae Textricella sp. 02

Mysmenidae

Genus 1 sp. 01

Mysmenidae

Genus 2 sp. 01

Nicodamidae Ambicodamus marae

Nicodamidae

Orsolobidae

Nicodamus maina

sp. 01

Oecobilidae

Oecobius naous

Onopidae

Gamasomoriha sp. 01

Oonopidae

Oonopidae

Oonopidae

Gamasomorpha sp. 13

Gamasomorpha sp. 02

Gamasomorpha sp. 03

Oonopidae

Gamasomorplus sp. 04

Oonopidae

Oonopidae

Oonopidae

Oonopidae

Oonopidae

Gamasomorpha sp. 05

Gamasomorpha sp. 06

Gamasomorpha sp. 07

Gamasomorpha sp.08

Oonopidae

Gamasomomha sp 09

Gamasomorpha sp. 10
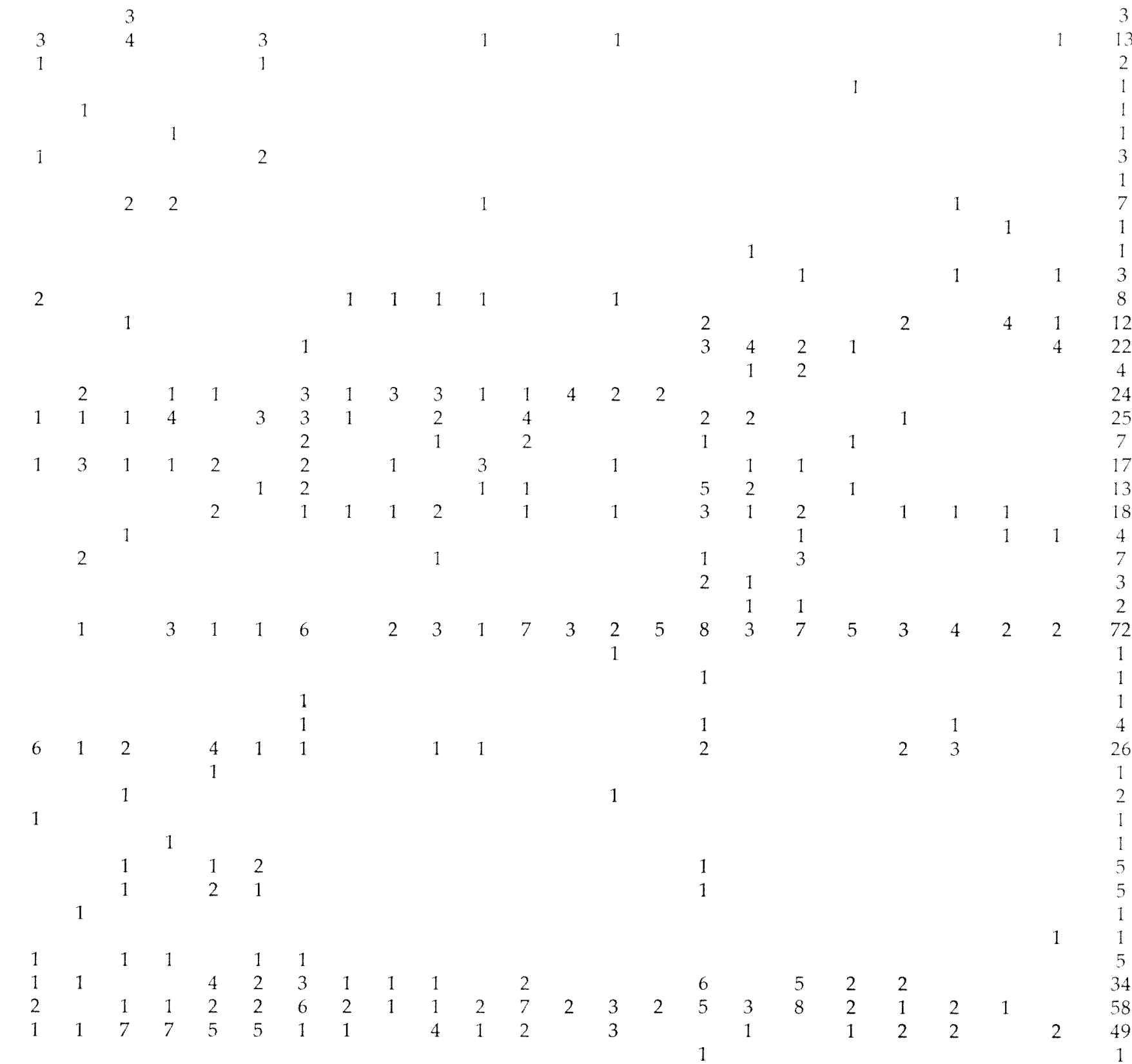


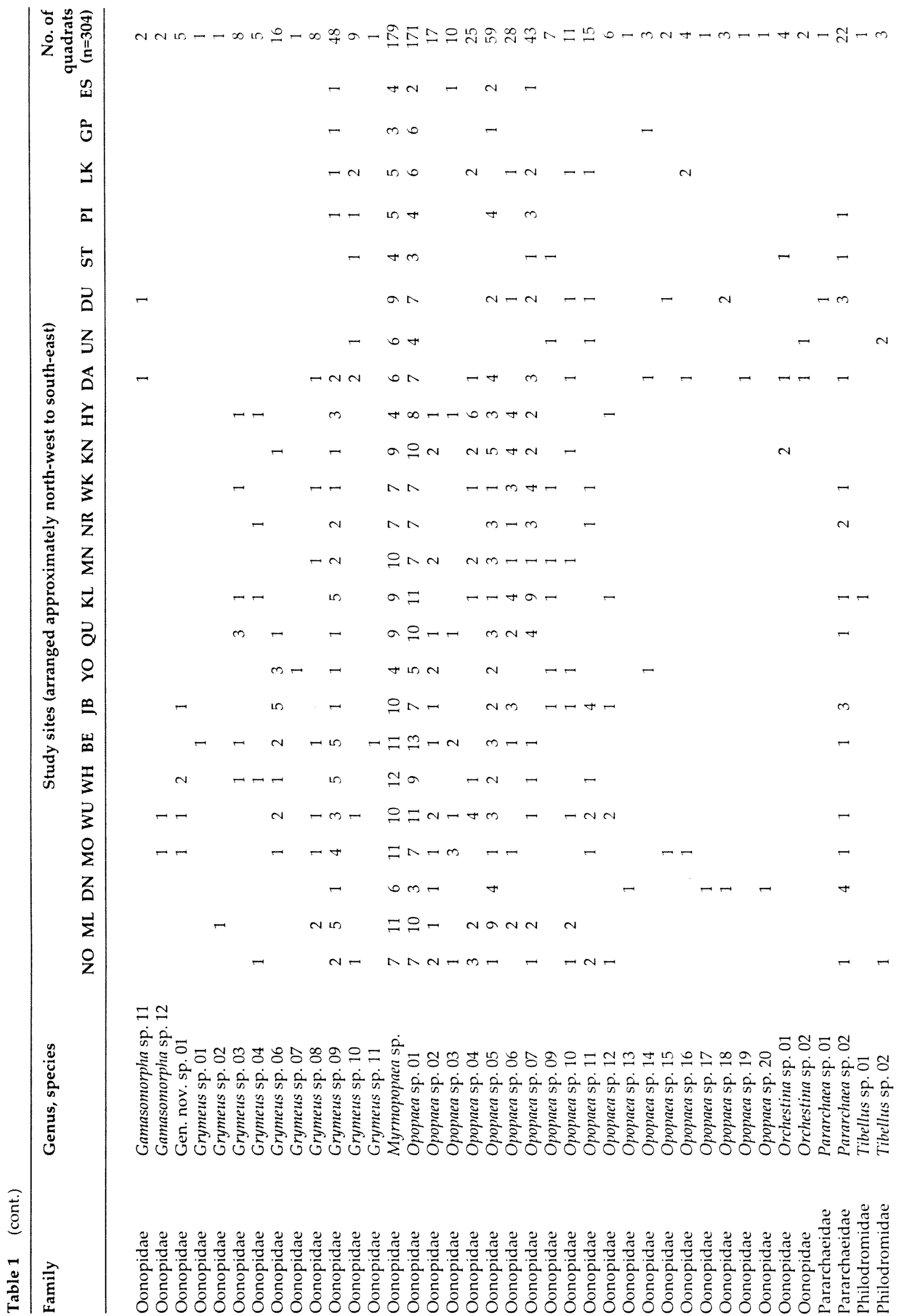


Bianor sp. 01

Salticidae

Breda jovialis

Salticidae

Salticidae

Salticidae

Salticidae

Clynotis sp. 01

Clynotis sp. 02

Clynotis sp. 03

Clynotis sp. 04

Salticidae

Salticidae

Salticidae

Salticidae

Clynotis sp. 05

Clynotis sp. 06

Clynotis sp. 07

Clynotis sp. 08

Clynotis sp. 09

Salticidae

Salticidae

Salticidae

Salticidae

Salticidae

Salticidae

Salticidae

Salticidae

Salticidae

Salticidae

Salticidae

Salticidae

Salticidae

Salticidae

Salticidae

Salticidae

Salticidae

Salticidae

Salticidae

Salticidae

Salticidae

Salticidae

Salticidae

Salticidae

Salticidae

Salticidae

Salticidae

Clynotis sp. 10

Clynotis viduus

Cytnea sp. 01

Damoetas sp. 01

Genus $1 \mathrm{sp} .01$

Genus $1 \mathrm{sp} .02$

Genus 1 sp. 03

Genus 1 sp. 04

Genus 1 sp. 05

Genus $1 \mathrm{sp} .06$

Genus 1 sp. 07

Genus $10 \mathrm{sp} .01$

Genus $12 \mathrm{sp} .01$

Genus 12 sp. 02

Genus $12 \mathrm{sp} .03$

Genus $12 \mathrm{sp} .04$

Genus 13 sp. 01

Genus 14 sp. 01

Genus 15 sp. 01

Genus 16 sp. 01

Genus 17 sp. 01

Genus $18 \mathrm{sp} .01$

Genus 19 sp. 01

Genus 2 sp. 01

Genus $20 \mathrm{sp} .01$

Genus 3 sp. 01

Genus 3 sp. 02

Salticidae 


NO ML DN MO WU WH BE JB YO QU KL MN NR WK KN HY DA UN DU ST PI LK GP ES (n=304)

\section{Salticidae}

Salticidae

Salticidae

Salticidae

Salticidae

Salticidae

Salticidae

Salticidae

Salticidae

Salticidae

Salticidae

Salticidae

Salticidae

Salticidae

Salticidae

Salticidae

Salticidae

Salticidae

Salticidae

Salticidae

Salticidae

Salticidae

Salticidae

Salticidae

Salticidae

Salticidae

Salticidae

Salticidae

Salticidae

Salticidae

Salticidae

Salticidae

Salticidae

Salticidae

Salticidae

Salticidae

Salticidae

Salticidae

Salticidae
Genus 3 sp. 03

Genus 3 sp. 04

Genus 3 sp. 05

Genus 3 sp. 06

Genus 5 sp. 01

Genus 6 sp. 01

Genus 7 sp. 01

Grayenulla australensis

Grayenulla nova

Holoplatys chudalupensis

Holoplatys dejongi

Holoplatys sp. 01

Holoplatys sp. 02

Holoplatys sp. 03

Hypoblemum sp. 01

Hypoblemum sp. 02

Lycidas chrysomelas

Lycidas michaelseni

Lycidas sp. 01

Lycidas sp. 02

Lycidas sp. 03

Lycidas sp. 04

Lycidas sp. 05

Lycidas sp. 06

Lycidas sp. 07

Lycidas sp. 08

Lycidas sp. 09

Lycidas sp. 10

Lycidas sp. 11

Lycidas sp. 12

Lycidas sp. 13

Lycidas sp. 14

Lycidas sp. 15

Lycidas sp. 16

Lycidas sp. 17

Lycidas sp. 18

Lycidas sp. 19

Lycidas sp. 20
1

1

$\begin{array}{lllllll}2 & 6 & 2 & 1 & 1 & & 2 \\ \end{array}$

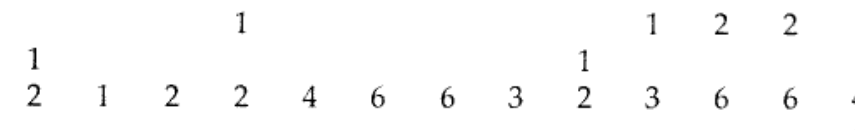

(1)

$\begin{array}{llllll}1 & 4 & 3 & 1 & 5 & 8 \\ 2 & 3 & 1 & 6 & 2 & 1\end{array}$

2

3

$\begin{array}{lll}1 & 3 & 6 \\ & & 1\end{array}$

2

$\begin{array}{llllll}1 & 1 & & 1 & 1 \\ & & 1 & & 1 \\ & & & & & 1\end{array}$

18

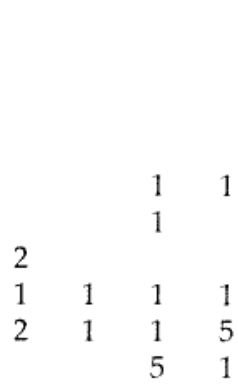

1

1

$\begin{array}{ll}9 & 6 \\ 1 & \\ 1 & 10 \\ 2 & 2 \\ 6 & \\ 1 & 1 \\ 1 & \\ 1 & \end{array}$

$\begin{array}{llcc}5 & 7 & 11 & 9\end{array}$

$\begin{array}{lll}9 & 1 & 2\end{array}$

$\begin{array}{lll}3 & 1 & 6 \\ & 1 & 2\end{array}$

1

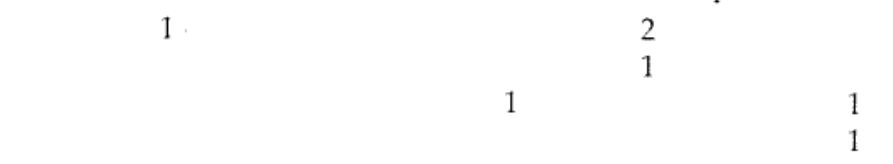

$\begin{array}{llll}1 & 1 & 3 & 2 \\ & 1 & & 2\end{array}$

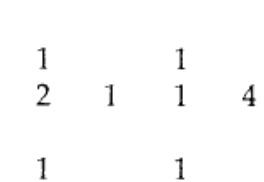

$\begin{array}{llll}2 & 1 & 1 & 3\end{array}$

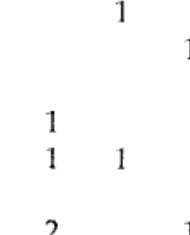

3

an

I

京

3

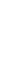


Salticidae

Salticidae

Salticidac

Salticidae

Salticidae

Salticidae

Salticidae

Salticidae

Salticidac

Salticidae

Salticidae

Salticidae

Salticidae

Salticidae

Salticidae

Salticidae

Salticidae

Salticidae

Salticidae

Salticidae

Salticidae

Salticidae

Salticidae

Salticidae

Salticidae

Salticidae

Salticidae

Salticidae

Salticidae

Salticidae

Salticidae

Salticidae

Salticidae

Salticidae

Salticidae

Salticidae

Salticidae

Salticidae

Segestriidae

Segestriidae

Segestriidae

Sparassidae

Sparassidae

Stiphidiidae

Stiphidiidae
Lucidas sp. 21

Lycidas sp. 22

Lycidas sp. 23

Lycidas sp. 24

Lycidas sp. 25

Lycidas sp. 26

Lycidas sp. 27

Maratus mungaich

Maratus pavonis

Maratus sp. 01

Maratus sp. 02

Maratus sp. 03

Maratus vespertilio

Margaromma sp. 0

Margaromma sp. 02

Myrmarachne sp. 01

Myrmarachne sp. 02

Ocrisiona leucocomis

Ocrisiona sp. 01

Opisthoncus sp. 01

Opisthoncus sp. 02

Opisthoncus sp. 03

Paraplatoides sp. 01

Prostheclina sp. 01

Seroaca sp. 01

Simaetha sp. 01

Simaetha sp. 02

Simaetha sp. 03

Simaethula sp. 01

Simaethula sp. 02

Simathula sp. 03

Sondra sp. 01

Zebraplatys fractioittato

Zebraplatys sp. 01

Zebraplatys sp. 02

Zebraplatys sp. 03

Zebraplatys sp. 04

Zenodorus sp. 01

Genus 1 sp. 01

Genus $1 \mathrm{sp} .02$

Genus 2 sp. 01

Delena sp. 0

Genus $1 \mathrm{sp} .01$

Baiami volucripes

Corasoides sp. 01
1

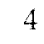

22

2

$\begin{array}{ll}1 & 1 \\ 1 & 1\end{array}$

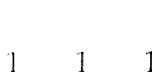

1

4

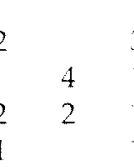

$\begin{array}{lll}3 & 4 & 2 \\ 3 & 3 & 5\end{array}$

$\begin{array}{llll}2 & & 1 & 1 \\ 3 & 4 & 1 & 1\end{array}$

21

$\begin{array}{llll}1 & & & \\ 1 & & & 1 \\ 1 & 1 & 1 & 2 \\ 1 & & 1 & \end{array}$

1

$\begin{array}{lll}1 & 1 & 1\end{array}$

$$
1
$$

2

1

1

1

1

1

1

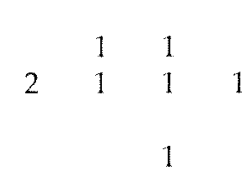

4

$\begin{array}{llllll} & 1 & 1 & 1 & & \\ 1 & 2 & & 3 & 4 & 1 \\ & 1 & & & & \end{array}$

11

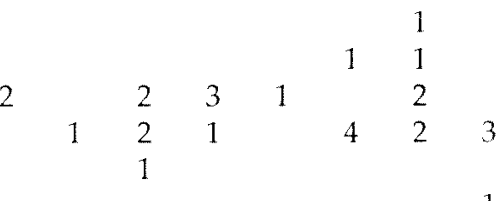

$\begin{array}{ll}3 & 1 \\ & 3\end{array}$

3 $\begin{array}{ll}1 & 3\end{array}$

2 $\begin{array}{ll}2 & 1 \\ & 1\end{array}$ 
Stiphidiidae Stiphidiidae Stiphidiidae Stiphidiidae Corasoides sp. 02 Corasoides sp. 03 Corasoides? sp. 04 Corasoides? sp. 05

Stiphidiidae Forsterina sp. 01

Tetrablemmidae Genus? sp ?

Tetragnathidae "metine" sp. 01

Tetragnathidae "metine" sp. 02

Theridiidae

sp. 01

Theridiidae

sp. 02

Theridiidae

Theridiidae

Theridiidae

Theridiidae

Theridiidae

Theridiidae

Theridiidae

Theridiidae

Theridiidae

Theridiidae

Theridiidae

Theridiidae

Theridiidae

Theridiidae

Theridiidae

Theridiidae

Theridiidae

Theridiidae

Theridiidae

Theridiidae

Theridiidae

Theridiidae

Theridiidae

Theridiidae

Theridiidae

Theridiidae

Theridiidae

Theridiidae sp. 02

sp. 04

sp. 05

sp. 06

sp. 07

sp. 13

"Ctenopalpus" "hirsti"

"Ctenopalpus" bicruciatus

"Genus A" sp.

"Nico" sp. 01

Ariamnes sp.

Enoplognatha sp.

Episinus sp. 01

Episinus? sp. 02

Dipoena sp. 01

Dipoena sp. 02

Euryopis sp. 01

Euryopis sp. 02

Euryopis sp. 03

Euryopis sp. 04

Euryopis sp. 05

Euryopis sp. 06

Euryopis sp. 07

Euryopis sp. 08

Euryopis sp. 09

Euryopis sp. 10

Euryopis sp. 11

Genus $1 \mathrm{sp} .01$

Genus 1 sp. 02

$\begin{array}{llllll}1 & 1 & & 1 & & \\ & 2 & 1 & & & \\ & 1 & 3 & 1 & 1 & 1 \\ & & & 1 & & 1\end{array}$

1

$\begin{array}{lll}4 & 2 & 1\end{array}$

$\begin{array}{ll}1 & 2 \\ & 1\end{array}$

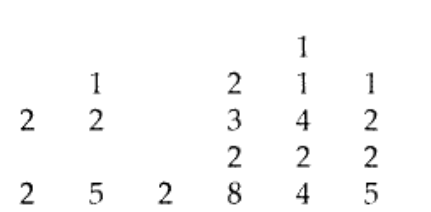

$\begin{array}{lllllll}3 & 2 & 1 & 2 & 3 & 1 & 1\end{array}$
1 
Theridiidae Theridiidae Theridiidae Theridiidae Theridiidae Theridiidae

Theridiidae

Theridiidae

Theridiidae

Theridida

Therididae

Theridiidae

Theridiidae

Theridiidae

Theridiidae

Theridiidae

Theridiidae

Theridiidae

Theridudae

Theridiidae

Theridiidae

Theridiidae

Theridiidae

Theridiidae

Theridiidae

Theridiidae

Theridiidae

Theriditalae

Theridiidae

Theridiidae

Theridiidae

Theridiidae

Theridiidae

Theridiidae

Theridiidae

Theridiidae

Theridiidae

Theridiidae

Theridiidae

Theridiidae

Theridiidae

Theridiidae

Theridiidae

Theridiidae

Theridiidae
Genus 1 sp. 03

Genus 1 sp. 04

Genus 2 sp. 01

Genus 2 sp. 02

Genus 3 sp. 01

Genus 3 sp. 02

Genus 4 sp. 01

Genus $4 \mathrm{sp} .02$

Genus 4 sp. 03

Genus 4 sp. 04

Genus $4 \mathrm{sp} .05$

Genus $4 \mathrm{sp} .06$

Genus $5 \mathrm{sp} .01$

Genus $5 \mathrm{sp} .02$

Genus 5 sp. 03

Genus 6 sp. 01

Genus 6 sp. 02

Genus 7 sp. 01

Genus 7 sp. 02

Genus? sp.

Gmogala sp. 01

Gmogala sp. 03

Gmogala sp. B

Gmogala sp. U

Hadrotarsinae Genus $1 \mathrm{sp} .01$

Hadrotarsinae Genus $2 \mathrm{sp} .01$

Hadrotarsinae Genus 3 sp. 01

Hadrotarsinae Genus D sp. 01

Hadrotarsinae Genus D sp. 02

Hadrotarsus sp. 02

Hadrotarsus sp. 03

Hadrotarsus sp. C

Latrodectus hasseltii

Phoroncidia sp. 01

Phoroncidia sp. 02

Phoroncidia sp. 03

Phoroncidia sp. 04

Phoroncidia sp. 05

Phoroncidia sp. 06

Steatoda sp. 01

Steatoda sp. 02

Steatoda sp. 03

Steatoda sp. 04

Steatoda sp. 05

Steatoda sp. 06

$\begin{array}{llllllllll}1 & 1 & & 1 & & 2 & 2 & 4 & & \\ & 1 & & & 1 & 1 & & & & \\ & & & & & & & & & \\ & 1 & 2 & 2 & & & 2 & & 1 & \\ & & 1 & & & & & & & 1\end{array}$

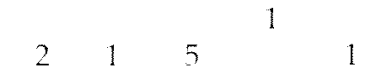

2

1

11

$\begin{array}{llll}13 & 5 & 12 & 1 \\ 13\end{array}$

$12 \quad 13$

1
2
1
5

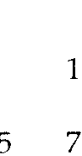

1
$1 \quad 2$
$7 \quad 1$
$7 \quad 4$

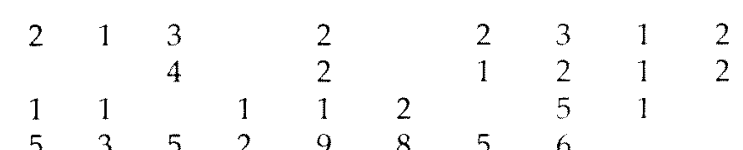

2

06

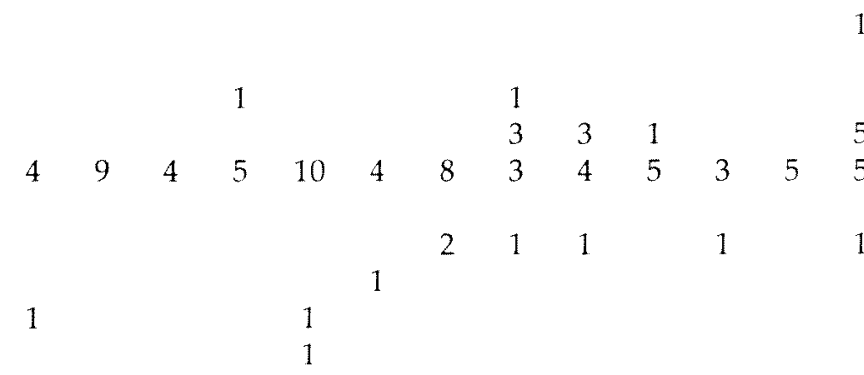

$$
1
$$

3


Theridiidae

Theridiidae

Theridiidae

Theridiidae

Thomisidae

Thomisidae

Thomisidae

Thomisidae

Thomisidae

Thomisidae

Thomisidae

Thomisidae

Thomisidae

Thomisidae

Thomisidae

Thomisidae

Thomisidae

Thomisidae

Thomisidae

Thomisidae

Thomisidae

Thomisidae

Thomisidae

Thomisidae

Thomisidae

Thomisidae

homisidae

Thomisidae

Thomisidae

Thomisidae

Thomisidae

Thomisidae

Thomisidae

Thomisidae

Thomisidae

Trochanteriidae

Trochanteriidae

Trochanteriidae

Trochanteriida
Steatoda sp. 07

Steatoda sp. 08

Steatoda sp. 09

Steatoda sp. B

Dinea sp.

Sidymella sp. 01

Sidymella sp. 02

Sidymella sp. 03

Sidymella sp. 04

Sidymella sp. 05

Sidymella sp. 06

Sidymella sp. 08

Sidymella sp. 09

Sidymella sp. 10

Sidymella sp. 11

Sidymella sp. 12

Stephanopis sp. 01

Stephanopis sp. 02

Stephanopis sp. 03

Stephanopis sp. 04

Stephanopis sp. 05

Stephanopis sp. 06

Stephanopis sp. 07

Tharpyna sp. 01

Tharpyna sp. 02

Tharpyna sp. 03

Tharpyna sp. 04

Tharpyna sp. 05

Tharpyna sp. 06

Tharpyna sp. 07

Tharpyna sp. 09

Tharpyna sp. 10

Tharpyna sp. 11

Tharpyna sp. 12

Tharpyna sp. 13

Rebilus sp. 01

Trachyspina madura

Trachyspina sp. 02

Trachyspina sp. 03

1

$\begin{array}{lll}2 & 2 & 1\end{array}$

$\begin{array}{lll}4 & 1 & 2\end{array}$

11

42

$\begin{array}{lll}1 & 1 \\ & & \\ & & \\ & 1 & \\ 1 & & \\ & & \\ 1 & & \end{array}$

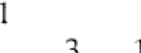

$\begin{array}{llllll}4 & 3 & 1 & 3 & 1 & 1\end{array}$

11

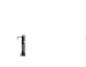

1

1

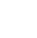

1

1

(

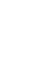


Trochanteriidae

Zodariidae

Zodariidae

Zodariidae

Zodariidae

Zodariidae

Zodariidae

Zodariidae

Zodariidae

Zodariidae

Zodariidae

Zodariidae

Zodariidae

Zodariidae

Zodariidae

Zodariidae

Zodariidae

Zodariidae

Zodariidae

Zodariidae

Zodariidae

Zodariidae

Zodariidae

Zodariidae

Zodariidae

Zodariidae

Zodariidae

Zodariidae

Zodariidae

Zodariidae

Zodariidae

Zodariidae

Zodariidae

Zodariidae

Zodariidae

Zodariidae

Zodariidae

Zodariidae

Zodariidae

Zodariidae

Zodariidae

Zodariidae

Zodariidae

Zodariidae

Zodariidae
Trachyspina sp. 04

Asteron-complex sp. 01

Asteron-complex sp. $02 \quad 2$

Asteron-complex sp. 03

Asteron-complex sp. 04

Asteron-complex sp. 05

Asteron-complex sp. 06

Asteron-complex sp. 07

Australutica quacrens

Australutica sp. 01

Australutica sp. 02

Australutica sp. 03

Cavasteron sp. 01

Chilumena reprobans

Chilumena sp. 01

Cyriocteasp. 0

Genus 1 sp. 01

Genus $1 \mathrm{sp} .02$

Genus $2 \mathrm{sp} .01$

Genus $3 \mathrm{sp} .01$

Genus 4 sp. 0

Habronestes australiensis

Habronestes grimuade

Habronestes sp. 01

Habranestes sp. 02

Habronestes sp. 03

Habronestes sp. 04

Habronestes sp. 05

Habronestes sp. 06

Habronestes sp. 07

Habronestes sp. 08

Habronestes sp. 09

Habronestes sp. 10

Habronestes sp. 11

Habronestes sp. 12

Habronestes sp. 13

Habronestes sp. 14

Habronestes sp. 15

Habronestes sp. 16

Habronestes sp. 17

Habronestes sp. 18

Habronestes sp. 19

Habronestes sp. 20

Habronestes sp. 21

Habronestes sp. 22

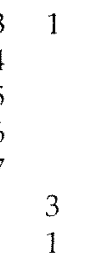

6

$\begin{array}{ll}3 & 4 \\ 1 & 3\end{array}$

656

(1)

$\begin{array}{lll}5 & 1 \\ 5 & 3\end{array}$

$\begin{array}{ll}1 & \\ 5 & 6\end{array}$

$\begin{array}{lll}6 & 7 & 4\end{array}$

1

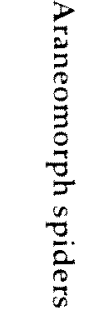

3

2

4
1
1

$\begin{array}{rl} & 4 \\ 1 & 1\end{array}$

$\begin{array}{r}2 \\ 1 \\ 1 \quad 2 \\ \hline\end{array}$

2
7
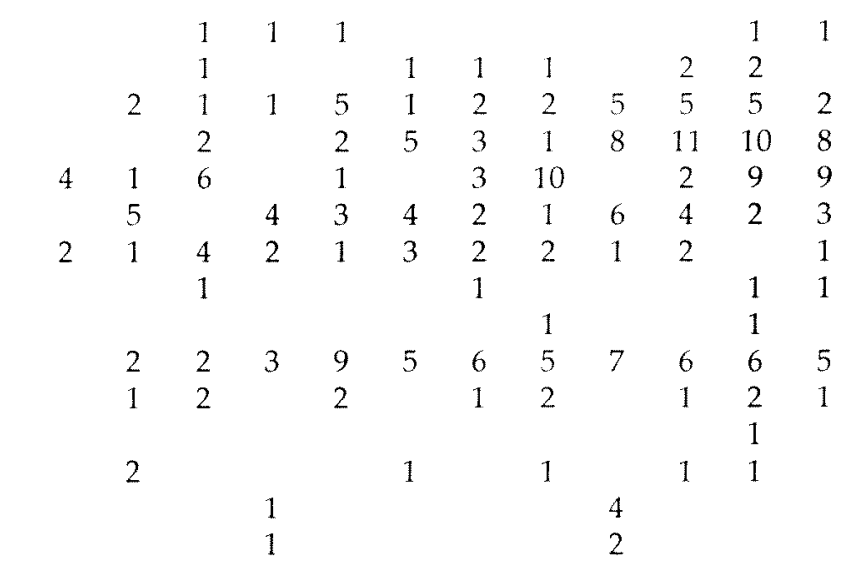

$\begin{array}{cc}11 & 2 \\ 1 & \end{array}$

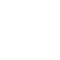


Zodariidae Zodariidae Zodariidae Zodariidae Zodariidae

Zodariidae

Zodariidae

Zodariidae

Zodariidae

Zodariidae

Zodariidae

Zodariidae

Zodariidae

Zodariidae

Zodariidae

Zodariidae

Zodariidae

Zodariidae

Zodariidae

Zodariidae

Zodariidae

Zodariidae

Zodariidae

Zodariidae

Zodariidae

Zodariidae

Zodariidae

Zodariidae

Zodariidae

Zodariidae

Zodariidae

Zodariidae

Zodariidae

Zodariidae

Zodariidae

Zodariidae

Zodariidae

Zodariidae

Zodariidae
Habronestes sp. 23

Habronestes sp. 24

Habronestes sp. 25

Habronestes sp. 26

Habronestes sp. 27

Habronestes sp. 28

Habronestes sp. 29

Habronestes sp. 30

Habronestes sp. 31

Habronestes sp. 32

Habronestes sp. 33

Habronestes sp. 34

Habronestes sp. 35

Habronestes sp. 36

Habronestes sp. 37

Habronestes sp. 38

Habronestes sp. 39

Heptasteron sp. 01

Hetaerica harveyi

Hetnerica sp. 01

Kerasteron sp. 01

Leptasteron sp. 01

Neostorena sp. 01

Neostorena sp. 02

Neostorena sp. 03

Neostorena sp. 04

Neostorena sp. 05

Neostorena sp. 06

Neostorena sp. 07

Neostorena sp. 08

Neostorena sp. 09

Neostorena sp. 10

Neostorena sp. 11

Neostorena sp. 12

Neostorena sp. 13

Neostorena sp. 14

Neostorena sp. 15

Neostorena sp. 16

Neostorena sp. 17
1

$\begin{array}{lllllllllll}1 & 1 & 3 & 2 & 2 & 1 & 1 & 3 & 4 & 1 & \end{array}$

2

4

$2 \quad 1$

29

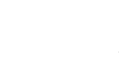
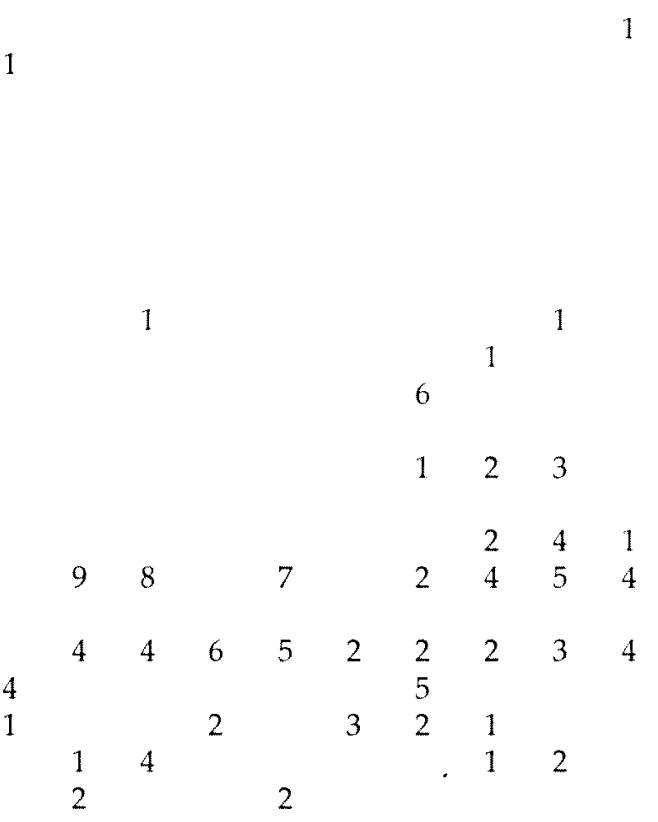

46

1

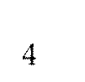

2

3

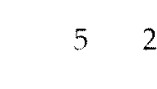

6
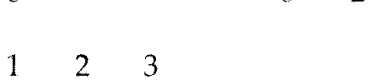

$1 \quad 1$

5

$\begin{array}{rlllllll} & 2 & 4 & 1 & 1 & 2 & & \\ 2 & 4 & 5 & 4 & & & 3 & 3 \\ 1 & \end{array}$

5

$\begin{array}{lllllllllll}7 & & 2 & 4 & 5 & 4 & & & 1 & & 1 \\ 5 & 2 & 2 & 2 & 3 & 4 & 2 & 4 & 1 & 5 & 2 \\ & & 5 & & & & 4 & 5 & 2 & 1 & 3\end{array}$

32

$\begin{array}{rr}2 & 3 \\ 1 & 1\end{array}$

$\begin{array}{ll}4 & 5 \\ & 1\end{array}$

2 


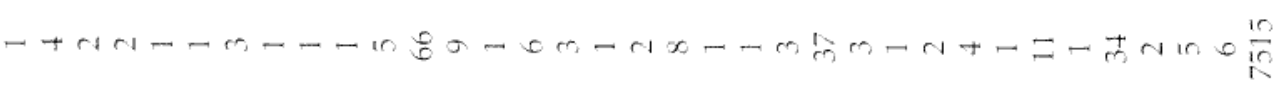

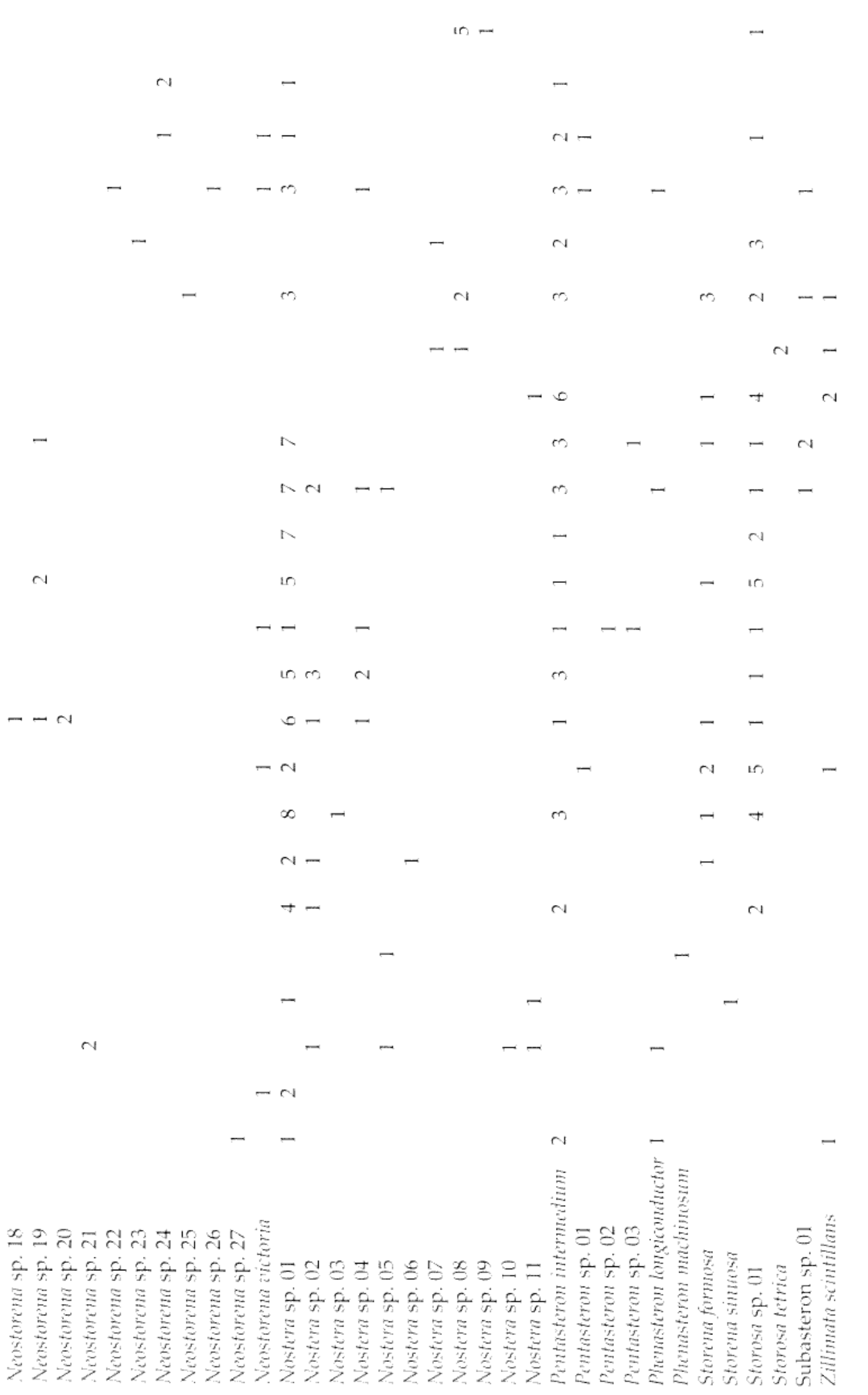

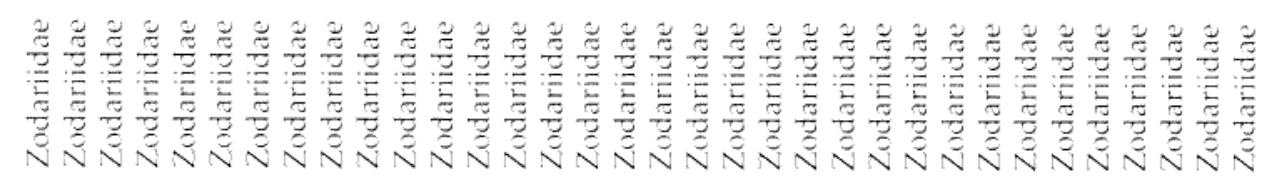


found during the survey, which all appear to differ from the species revised by Platnick and Forster (1989): Chasmocephalon sp. 1 (DA, JB, UN and YO), Chasmocephalon sp. 2 (DA, JB, NR and UN) and Chasmocephalon sp. 3 (DA, ST and UN). Anapids were generally absent from the northern and eastern quadrats and were more abundant in the southern quadrats.

\section{Araneidae}

We recorded a variety of araneids, but usually in very low numbers. The majority of araneid species are arboreal and, because they are not reliably captured in pitfall traps, were excluded from the quantative analyses.

\section{Cyatholipidae}

The family Cyatholipidae occurs in the majority of the southern continents (Forster et al., 1988; Griswold, 2001) and the Australian fauna is represented by only a few named genera. The softbodied genera such as Teemenaarus, Tekellatus and Toddiana are generally restricted to temperate woodland or rainforest habitats, while members of the genus Matilda occur throughout mainland Australia usually in open woodland habitats. Harvey et al. (2000) found three species of Matilda in the Carnarvon Basin region, and this study found two species of this widespread and abundant genus.

\section{Cycloctenidae}

Cycloctenid spiders are restricted to Australia and New Zealand and are vagrant hunters occurring in leaf litter or under stones and rocks. The Western Australian fauna consists of small spiders of uncertain generic affinity. In this survey, Genus 1 sp. 1 was found once at DA and Genus 2 sp. 1 was found once at LK.

\section{Deinopidae}

Members of the cosmopolitan family Deinopidae construct spectacular webs that they use to ensnare their prey (Raven et al., 2002). A single species of Deinopis was found sporadically throughout the study area at 10 quadrats. Most deinopids are generally arboreal and, because they are not reliably captured in pitfall traps, were excluded from the quantative analyses.

\section{Desidae}

Spiders of the family Desidae are commonly found in a wide variety of Western Australian terrestrial habitats. This survey recorded 39 species, the majority of which were found in few quadrats.

\section{Filistatidae}

Filistatid spiders are found in a variety of regions in mainland Australia, and are represented by several species of Wandella and by a single cavedwelling species of Yardiella (Gray, 1994). Most species of Wandella occur under the bark of trees, although Harvey et al. (2000) recorded five species in pitfall traps in the Carnarvon Basin region indicating that some species either live permanently on ground habitats (such as in litter or under stones) or that they move from tree trunks onto the ground at certain times of the year. The present survey recorded Wandella barbarella Gray.

\section{Gallieniellidae}

The Australian members of the gnaphosoid family Gallieniellidae were recently revised by (Platnick, 2002) who found a small fauna of five genera and 27 species. Harvey et al. (2000) encountered five species in the Carnarvon survey, including two species of Meedo. The present survey recorded six species, including Meedo houstoni Main which was also found in the Carnarvon survey.

\section{Hahniidae}

Hahniids are tiny litter or bark dwelling spiders. Ten species were found in low numbers during the present survey, while no hahniids were found in the Carnarvon survey (Harvey et al., 2000).

\section{Hersiliidae}

Hersiliid spiders are generally found on tree trunks where they lie cryptically in wait for their prey. Baehr and Baehr (1987, 1989, 1992, 1993, 1995, 1998) have recorded a total of 25 species of Tamopsis from Western Australia, with a further four species of Hersilia from northern Western Australia. Nine species of Tamopsis were recorded in this survey, most from just a handful of localities, suggesting that they are infrequently captured in pitfall traps due to their arboreal lifestyle. For this reason the family was excluded from the quantative analyses.

\section{Lamponidae}

The Lamponidae are restricted to Australia, New Caledonia and New Guinea, with two species accidentally introduced into New Zealand; the vast majority of lamponids are Australian (Platnick, 2000). The Lamponidae were revised by Platnick (2000) who recognised numerous Australian genera species.

During this study 41 species in 13 genera encompassing all three subfamilies were identified. The Lamponinae was represented by six genera, Lampona, Lamponata, Lamponega, Lamponella, Lamponina and Lamponusa, and 26 species, of which 17 were Lampona species. The Centrothelinae were represented by six genera, Asadipus, Bigenditia, Longepi, Notsodipus, Prionosternum and Qucenvic, and 14 species. The Pseudolamponinae were represented by Pseudolampona bore Platnick. The 
genera Lamponella, Prionostemum and Queenvic were restricted to the southern quadrats.

\section{Linyphiidae}

Linyphiids were abundant at many sites, but were excluded from the quantitative analyses because of uncertainties in associating male and female specimens and in interpreting the taxonomic significance of slight morphological differences in the structure of the male and female genitalia. The family is largely unstudied in Australia and remains an enormous challenge in ecological analyses.

\section{Liocranidae}

Liocranid spiders are small to medium sized hunting spiders that are moderately diverse in the Australian region. We recorded nine species, and only sp. 12 was found at more than 10 sites.

\section{Lycosidae}

The spider family Lycosidae is a diverse group that is poorly known in Australia. The genera Artoria and Venatrix have been recently revised (Framenau and Vink, 2001; Framenau, 2002) but the remaining genera are still in flux. The vast majority of the Australian wolf spider fauna has been attributed to the genus Lycosa but these species bear little affinity to the type species of Lycosa (Vink et al, 2002). The systematics of the Australian wolf spiders is currently being revised (Framenau, pers. comm.). We identified 61 lycosid species in the present survey, but the case for some of the identifications is tenuous and the fauna may be smaller.

\section{Micropholcommatidae}

The tiny spiders comprising the family Micropholcommatidae are abundant in Australian forests but have largely remained unstudied except for the work of Hickman (1944, 1945, 1979) and Forster (1959). We found several species tentatively placed in the genus Micropholcomma.

\section{Mysmenidae}

The tiny spiders of the Mysmenidae are only rarely collected in pitfall traps, and only two species were found in this survey, each from a single site.

\section{Nicodamidae}

The Nicodamidae were revised by Harvey (1995) who found two genera in New Zealand and five genera in Australia, one of which also occurs in New Guinea. The Western Australian fauna is represented by three species, Nicodamus mainae Harvey, Ambicodamus marae Harvey and A. kochi Harvey. Nicodamus mainae was found to be widespread throughout the survey area, which is consistent with the broad distributional range recorded by Harvey (1995). Ambicodamus marae was only found at DA07, JB10 and LK12, each representing range extensions for this species which was previously found to be restricted to high rainfall areas, predominately in the karri forests of the south coast (Harvey, 1995) and in outlying populations in the Darling Range (Brennan, 1999). Ambicodamus kochi was found at NO11.

\section{Oecobiidae}

The spider family Oecobiidae is represented in the Australian region by introduced species (see Santos and Gonzaga, 2003), and this study found Oecobius navus at several sites. Unfortunately this species was coded as "Annulipes sp. 1" and "Annulipes sp. 2" in the analysis.

\section{Oonopidae}

The tiny spiders of the family Oonopidae are an extremely abundant and diverse component of the Australian fauna, and we found 46 species in a variety of genera including Gamasomorpha, Grymeus, Mymopopaea, Opopaea, Orchestina and a new genus.

\section{Orsolobidae}

The Gondwanan family Orsolobidae is represented in the Australian fauna by members of several genera, of which Tasmanoonops and Australobus have been found in Western Australia (Forster and Platnick, 1985). Further unnamed species of both genera are known (Harvey, unpublished data). A single specimen of an unnamed species was found at WH02.

\section{Pararchaeidae}

The family Pararchaeidae is restricted to Australia and New Zealand where they occur in forested and woodland habitats. Two unnamed species of Pararchaea were found in the survey. Pararchaea sp. 1 was found at one quadrat whilst Pararchaea sp. 2 was found at a variety of sites throughout the survey area.

\section{Philodromidae}

The Australian philodromid fauna is small (Raven et al., 2002) with just a handful of recognised species in two genera, Philodromus and Tibellus. We recognised four species of Tibellus in the present survey, each collected in very low numbers, presumably due to their primarily arboreal preferences.

\section{Salticidae}

The Salticidae are the largest spider family (Platnick, 2004) and the Australian fauna is imperfectly known (Davies and Żabka, 1989). We recorded 121 species in 41 genera, of which the vast 
majority are undescribed. The Salticidae were the subject of a separate analysis in this volume (Guthrie and Waldock, 2004).

\section{Segestriidae}

Only three species of Segestriidae were recognised, in contrast to the seven species found in the Carnarvon survey.

\section{Stiphidiidae}

The Australasian spider family Stiphidiidae was represented in the survey by a single species of Baiami, three species of Corasoides, a species of Forsterina, and two species tentatively placed in Corasoides.

\section{Tetrablemmidae}

The spider family Tetrablemmidae is currently represented within Australia by a single named species, Tetrablemma okei from Victoria, but numerous unnamed species occur in eastern and northern Australia (Harvey, unpublished data). A single female tetrablemmid was collected at MOO2, which represents the first record of this family from southern Western Australia.

\section{Tetragnathidae}

Tetragnathid spiders are represented in southwestern Australia by several genera such as Nephila and Tetragnatha, as well as a series of poorly known metines. This survey found two such metine species in low numbers.

\section{Theridiidae}

Theridiid spiders are a ubiquitous part of the spider fauna of most regions of the world, and we recorded 80 species.

\section{Thomisidae}

We recorded 31 species of Thomisidae during the survey, primarily in the genera Sidymella, Stephanopis and Tharpyna. As the majority of these species are considered to be arboreal or corticolous and, because they are not reliably captured in pitfall traps, they were excluded from the quantative analyses.

\section{Trochanteriidae}

Platnick (2002) has recorded a rich trochanteriid fauna in Australia, represented by 14 indigenous genera. We found five species of this family, including four species of Trachyspina and a single species of Rebilus, each from a single quadrat.

\section{Zodariidae}

Recent systematic research on the Australian zodariid fauna has revealed a highly diverse fauna consisting of numerous genera (e.g. Jocqué and Baehr, 1992, 2001; Jocqué, 1995a,b; Baehr, 2003a,b, in press; Baehr and Jocqué, 2000, 2001; Jocqué and Baehr, 2001; Baehr and Churchill, 2003;). The 117 species of Zodariidae recorded during the survey were the subject of a separate analysis in this volume (Durrant, 2004), in which there was no discernible relationships between species composition and substrate data.

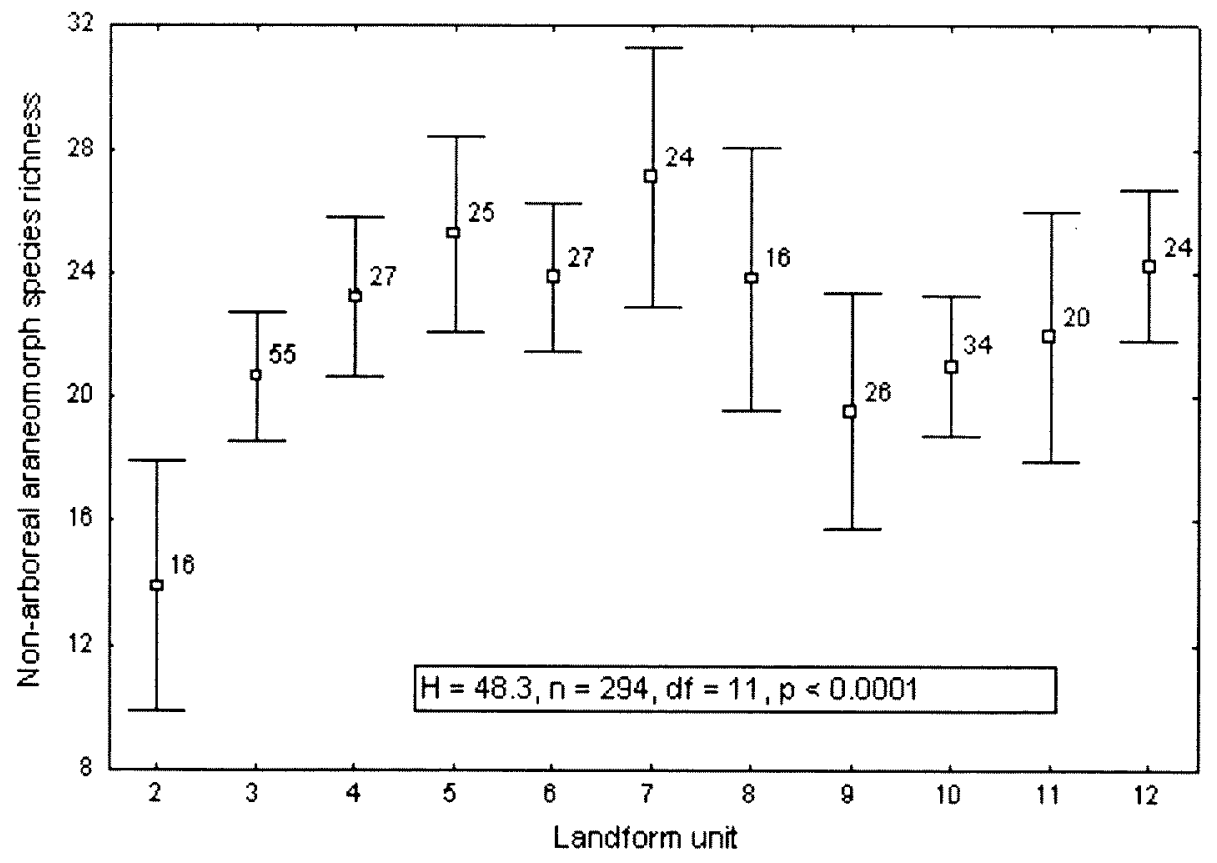

Figure 3 Relationship between quadrat species richness and landform unit. Mean and $95 \%$ confidence intervals are displayed. Means are labelled with the number of quadrats sampled in each landform. $\mathrm{H}=\mathrm{Kruskall}-$ Wallis coefficient, $\mathrm{n}=$ number of quadrats, $\mathrm{df}=$ degrees of freedom, and $P=$ probability. Because of trap-flooding problems, data from landform unit 1 (freshwater swamps) are excluded. 


\section{Richness}

A total of 622 ground-dwelling araneomorph spider species were recorded from the 304 quadrats, an average of 21.9 species per quadrat (s.d. $=8.3$ ). Figure 3 provides a graphical representation of species richness within each landform unit. Natural saltflats (unit 2) had the fewest species, while dissection valley slopes (units 4 to 8 ) and duricrust pavements (unit 12) were the richest.

The relationship between salinity risk (SAL, as a measure of secondary salinity) and species richness was quantified for the ground-dwelling araneomprphs overall, and for the three most speciose families separately: Salticidae (121 species), Zodariidae (117) and Lycosidae (61). Together, these comprised $48 \%$ of the araneomorph species recorded during the survey. This analysis was restricted to the 55 quadrats sampled on landform unit 3 (dissection valley floors), the unit with sufficient quadrats sampled in each of the four salinity risk categories to offer a balanced comparison (McKenzie et al, 2003). Together the three families analysed comprised $47 \%$ of the araneomorph richness recorded on landform unit 3 . Salticids and zodarids showed a significant negative correlation with electrical conductivity, while lycosids showed a significant positive correlation (Kendals Tau $=-0.26, p=0.006 ; \tau=-0.37$, $P=0.0001 ; \tau=0.31, P=0.001$, respectively). A significant negative correlation was also found when all araneomorph families, except lycosidae, were included $(\tau=-0.27, P=0.004)$. Although richness was tightly correlated with numer of specimens $(\tau=0.56, P<0.0001)$, the latter was not significantly correlated with salinity risk. This suggests that the significance of the salinity risk correlation was related to species richness rather than being an artefact of the relatively low numbers of identified specimens per quadrat $(87, \mathrm{~s} . \mathrm{d} .=60, \mathrm{n}$ $=55$ ). Scatter-plots of species richness versus EC showed that individual outlying points did not determine the significance of these correlations. Using a larger data-set of arachnids and vertebrates that encompassed the araneomorph data being investigated in this paper, McKenzie et al. (2003) showed that these secondarily salt-affected quadrats held a species-poor sub-set of the assemblages recorded on their unaffected counterparts, as well as a few natural salt-flat species.

\section{Singleton species and local endemism}

One hundred and eighty-five species of araneomorph spiders were recorded at only one quadrat, representing $29.7 \%$ of the entire data-set.

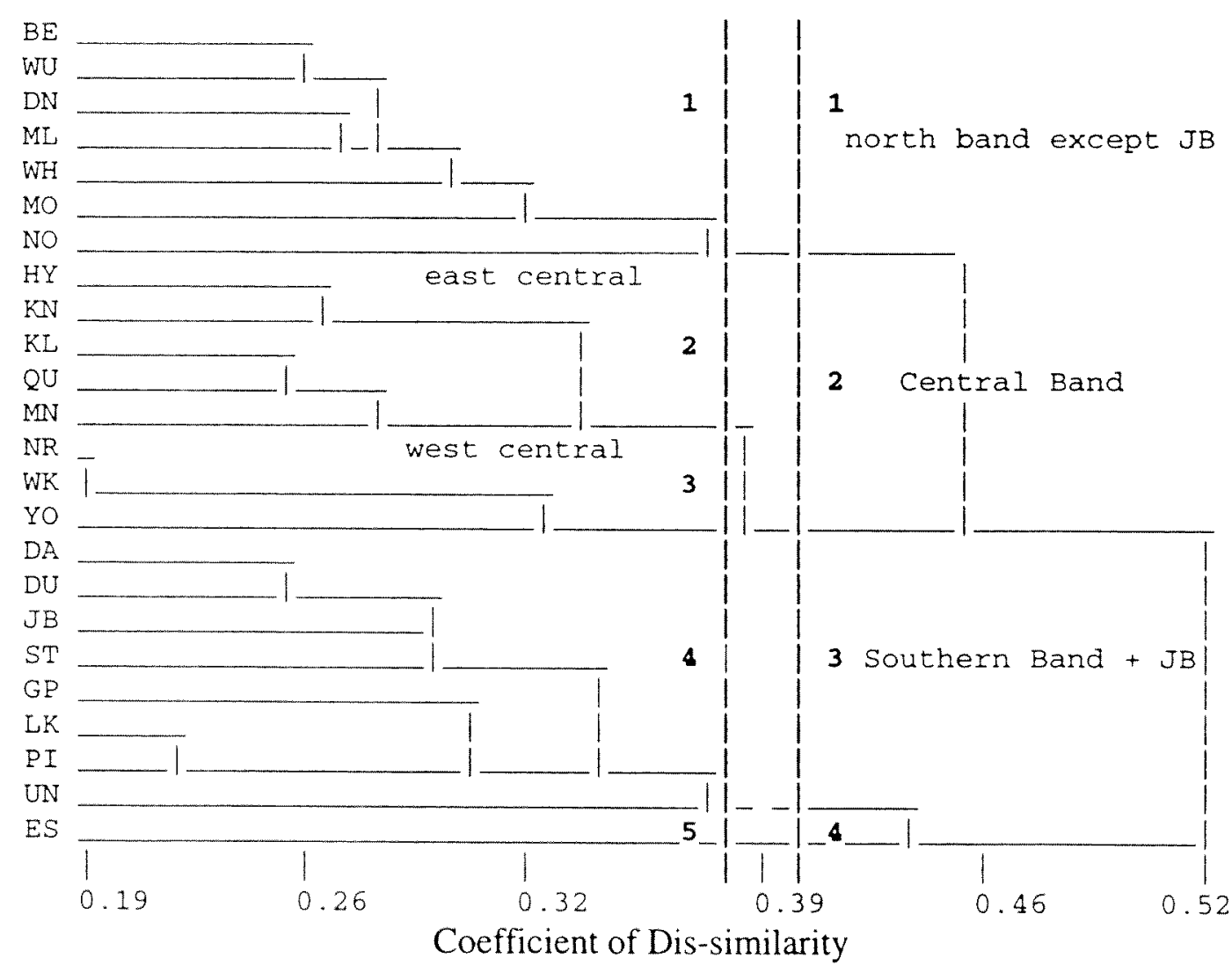

Figure 4 Dendrogram structure derived by classifying the 24 survey areas according to the combined genera composition of their component quadrats (a total of 166 non-arboreal araneomorph genera). Singleton species were retained in the 304 quadrat versus 622 species matrix from which the survey area genera lists were derived. Genera that were recorded in only a single survey area were included in the analysis. 


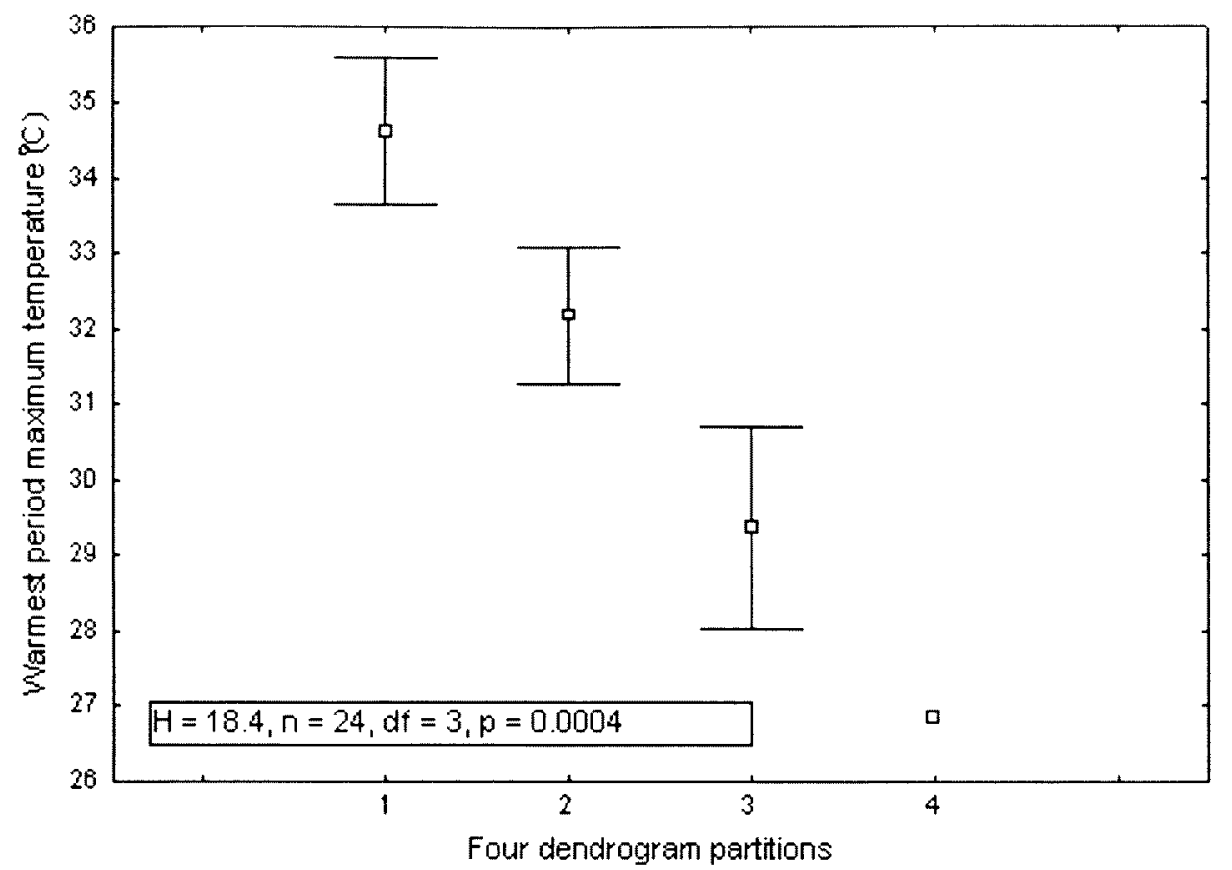

Figure 5 The four partitions in Figure 4 were most clearly separated by warmest period maximum temperature. Mean and $95 \%$ confidence intervals are based on climatic values averaged across the 12 to 13 quadrats within each survey area $(\mathrm{H}=$ Kruskall-Wallis coefficient, $\mathrm{n}=$ number of quadrats, $\mathrm{df}=$ degrees of freedom, and $P=$ probability).

On average, each quadrat had only one singleton (sd $=1.01, n=304$ ). Of the 400 non-singleton species recorded on the 240 quadrats that were both unaffected by secondary salinity and adequately sampled, 38\% (152/400) were more geographically localised than would be expected by chance alone $(P<0.05)$, compared to 1000 trials using an equivalent number of randomly arrayed records (quadrat intersections), randomly arrayed, for each species. Overall, $65 \%$ of species $(261 / 400)$ were recorded at between 2 and 10 quadrats and $26 \%$ of these (67/261) were more geographically localised than would be expected by chance $(P<0.05)$. These 67 species were recorded in an average of 3.3 survey areas (s.d. $=1.9$ ), and most were recorded only in the same or adjacent survey areas (51/67 species). This should not be interpreted as unambiguously implying that all 67 species are locally endemic. Records of 66 of the 67 species extended into survey areas on the periphery of the study area and further survey work will probably show that many of these species extend beyond this periphery. Because of the elongate shape of the study area (Figure 1), 18 of the 24 survey areas were peripheral.

\section{Patterns in genus composition}

By classifying survey areas in terms of their genera composition we could minimise the effects of species endemism and substrate, and reduce the influence of study area edge effects and sampling noise, on the partition structure of the resulting dendrogram. The compositional pattern, as summarised by the resulting dendrogram structure at the four partition-level (Figure 4), was closest to the warmest period maximum temperature (see Figure 5). At the five partition-level significant separation of all partitions was best achieved by a combination of temperature diurnal range and warmest quarter mean temperature (Table 2). Together, these two climatic attributes yielded a climatic topography and axis only slightly different from warmest period maximum temperature.

To see whether substrate attributes emerged as significant correlates with compositional patterns in araneomorph genera, the 240 quadrats were classified in terms of similarities in the presence and absence of their 162 araneomorph genera. The four genera that occurred at only a single quadrat are excluded from this analysis. The resulting dendrogram structure could be interpreted down to the seven partition-level, where six of the seven groups could be separated statistically using a combination of four quadrat attributes: SAL (salinity risk), soil $\mathrm{pH}$, warmest quarter mean temperature and precipitation seasonality (Table 2). Group 5 could not be separated uniquely, but comprised just three of the 240 quadrats.

\section{Patterns in species composition}

Figure 6 is the dendrogram structure derived when the survey areas were classified according to their total araneomorph species composition. This dendrogram reveals a strong geographical pattern related to rainfall and temperature gradients. When the structure was examined statistically against survey area climatic attributes, the tightest 


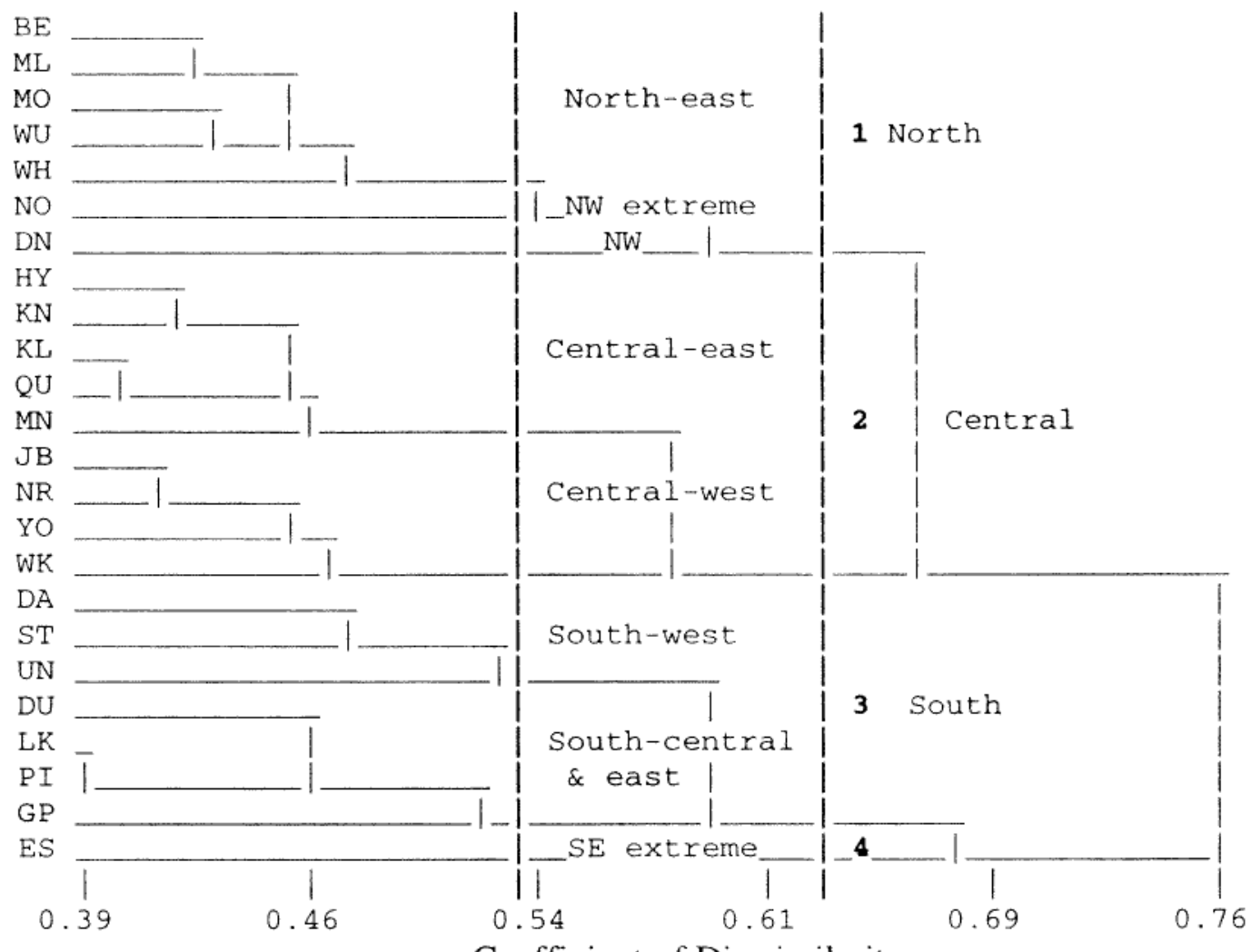

Coefficient of Dis-similarity

Figure 6 Dendrogram structure derived by classifying the 24 survey areas according to the combined species composition of their component quadrats. Singleton species were retained in the 304 quadrat versus 622 species matrix from which the survey area species lists were derived.

relationship was with warmest period maximum temperature (Table 2), a north-north-east to southsouth-west axis related to the hotter summers in the north-north-east (Figure 7).

When the 240 quadrats were classified in terms of similarities in their species composition, the dendrogram could be interpreted down to the eight-partition level (Figure 8). All partitions could be separated statistically using a combination of four quadrat attributes: soil salinity, soil pH,

Table 2 Environmental attributes that best separated the classification partition structures derived from the four compositional analyses $(\mathrm{A}=$ survey areas, $\mathrm{G}=$ genera, $\mathrm{Q}=$ quadrats, $\mathrm{S}=$ species, $\mathrm{H}=$ Kruskall-Wallis coefficient, $P=$ probability, $\mathrm{m} \times \mathrm{Twm} \mathrm{P}=$ warmest period maximum temperature ${ }^{\circ} \mathrm{C}, \mathrm{Tdir}=$ temperature diurnal range, $\mathrm{Tw} \mathrm{mQ}=$ warmest quarter mean temperature, $\mathrm{Psea}=$ precipitation seasonality $\mathrm{mm}, \mathrm{SAL}=$ salinity risk, $\mathrm{EC}=$ electrical conductivity $\mathrm{mS} / \mathrm{m}, \mathrm{pH}=$ soil $\mathrm{pH}$ ).

\begin{tabular}{|c|c|c|c|c|c|}
\hline Data matrix & $\begin{array}{l}\text { Dendrogram } \\
\text { Partition Level }\end{array}$ & $\begin{array}{l}\text { Physical } \\
\text { Attribute }\end{array}$ & Partitions Separated & $\mathrm{H}(P)$ & Comment \\
\hline $24 \mathrm{~A} \times 166 \mathrm{G}$ & $\begin{array}{l}4 \text { (Figure } 4) \\
5 \text { (Figure } 4)\end{array}$ & $\begin{array}{l}\text { mxTwmP } \\
\text { Tdir } \\
\text { TwmQ }\end{array}$ & $\begin{array}{l}\text { all } \\
1 \& 2 \text { vs } 3 \text { vs } 4 \text { vs } 5 \\
1 \text { vs } 2\end{array}$ & $\begin{array}{l}18(0.0004) \\
17(0.002) \\
20(0.0006)\end{array}$ & Figure 5 \\
\hline $24 \mathrm{~A} \times 622 \mathrm{~S}$ & 4 (Figure 6) & mxTwmp & all & $20(0.0002)$ & \\
\hline $\begin{array}{l}240 \mathrm{Q} \times 162 \mathrm{G} \text {, } \\
\text { excluding } \\
\text { singletons }\end{array}$ & 7 & $\begin{array}{l}\text { SAL } \\
\text { PH } \\
\text { TwmQ } \\
\text { Psea }\end{array}$ & $\begin{array}{l}1-5 \text { is } 6 \& 7 \\
6 \text { vs } 7 \\
1 \text { is } 2 \& 3 \text { vs } 4 \\
2 \text { is } 3\end{array}$ & $\begin{array}{l}31(0.0001) \\
28(0.0001) \\
137(0.00001) \\
38(0.0001)\end{array}$ & $\begin{array}{l}\text { Partition } 5 \text { not separated } \\
\text { from 1-4 }\end{array}$ \\
\hline $\begin{array}{l}240 Q \times 4005, \\
\text { excluding } \\
\text { singletons }\end{array}$ & 8 (Figure 8$)$ & $\begin{array}{l}\mathrm{EC} \\
\mathrm{pH} \\
\text { TwmQ } \\
\text { Psea }\end{array}$ & $\begin{array}{l}1-6 \text { vs } 7 \& 8 \\
1-5 \text { vs } 6 \text { vs } 7 \text { os } 8 \\
1 \& 3 \text { is } 2 \text { vs } 4 \text { is } 5 \text {; } 6 \text { vs } 7 \text { is } 8 \\
1 \text { vs } 3\end{array}$ & $\begin{array}{l}37(0.000001) \\
35(0.00001) \\
147(0.0000) \\
56(0.00000)\end{array}$ & \\
\hline
\end{tabular}




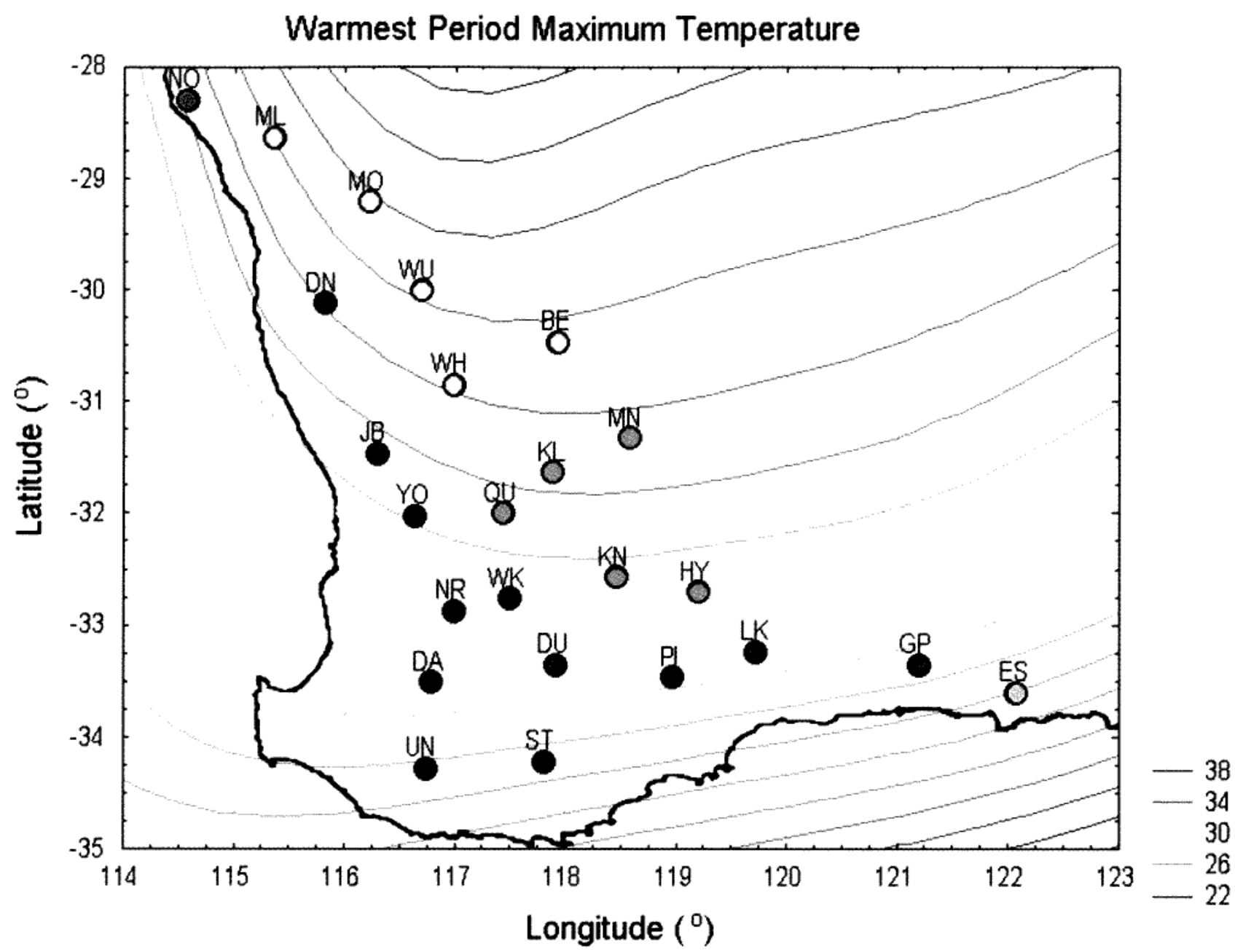

Figure 7 Results of the survey area classification (Figure 6) displayed geographically. Warmest period maximum temperature contours $\left({ }^{\circ} \mathrm{C}\right)$ are superimposed on the survey area map. Colours indicate relationships down to the eight partition-level in the classification dendrogram and are ordered from yellow to blue down the dendrogram (Figure 6). Highest temperature values are in the north.

warmest quarter mean temperature and rainfall seasonality (Figure 8, Table 2).

Warmest quarter mean temperature is tightly intercorrelated with warmest period maximum temperature (Kendall's Tau $=0.92, p<0.001$ ). Thus, the four classification analyses yielded similar results (Table 2), although salinity emerged as an important factor in the two quadrat classifications (either as salinity risk or as soil electrical conductivity), along with soil $\mathrm{pH}$.

\section{DISCUSSION}

The spider fauna of southern Western Australia consists of rich assemblages (Brennan and Majer, in press; Brennan et al., in press), with numerous genera and species showing varying degrees of regional endemicity. Knowledge of the detailed taxonomy and distributions of this fauna is restricted to just a few families (see Introduction). Many of these studies show that whilst species endemism in southern Western Australia is high, there is also a considerable component of the fauna that ranges into other areas of southern Australia or into the central or northern regions of Western Australia. Regrettably, many other families are taxonomically poorly known and the identifications made during this study cannot be readily correlated with the faunae found in other regions of the country.

There is limited extrinsic knowledge available on patterning in Western Australia araneomorph spider communities, which precluded a multivariate statistical approach to the analysis. Relationships revealed by such an analysis could be artefacts of sampling design. Also, many of the environmental attributes recorded for each study quadrat were strongly intercorrelated. Thus, to generate hypotheses for future studies we restricted our analyses to a univariate approach.

Overall araneomorph diversity patterns across the wheatbelt study area conformed most closely to a combination of soil salinity, a summer mean temperature gradient with a north-south axis, and a precipitation seasonality gradient with an east-west 
BEOI-5,7-9,12,13, DNO1,2,6,8

KN10, MLO1-13, MN03,4,7,10,

MOO1,3,5,6,9-12, NOOl-7,10,11

PI05, QU04, WH04,5,7,8,11,13

WU01-6,9-12, YO02, 10

DN05, DU02-4,9-13, ES07,9

GP01-4,6,12, HY01,3-7,9,10

JB01,2,5-10,12,13, KL03-12

KNO2-6,11, LK02,4-6,8,10-13

MN01,5,6,11, NRO1-4,6,8-12

PI01,6-9,12, QU01-3,6,9-12

ST09,10, WH03,6,9,10,

WK01,6-11,13, YO01,4-8,12,13

DN03,4,9-12

DA01,3,4,6,8-11,13, DU01

GP08,9, LK07, NR05,7

ST01,6,13, UN03, YOO3

DA12, ESO1,2,5,8,11

GP13, PI13, ST02-5,7

UNO1,2,5-7,9-12

GP10, PI11

BE11, DU05, ES06, HY02, KL01, LK03, ML12, MN09, MO04, ST08, WU08

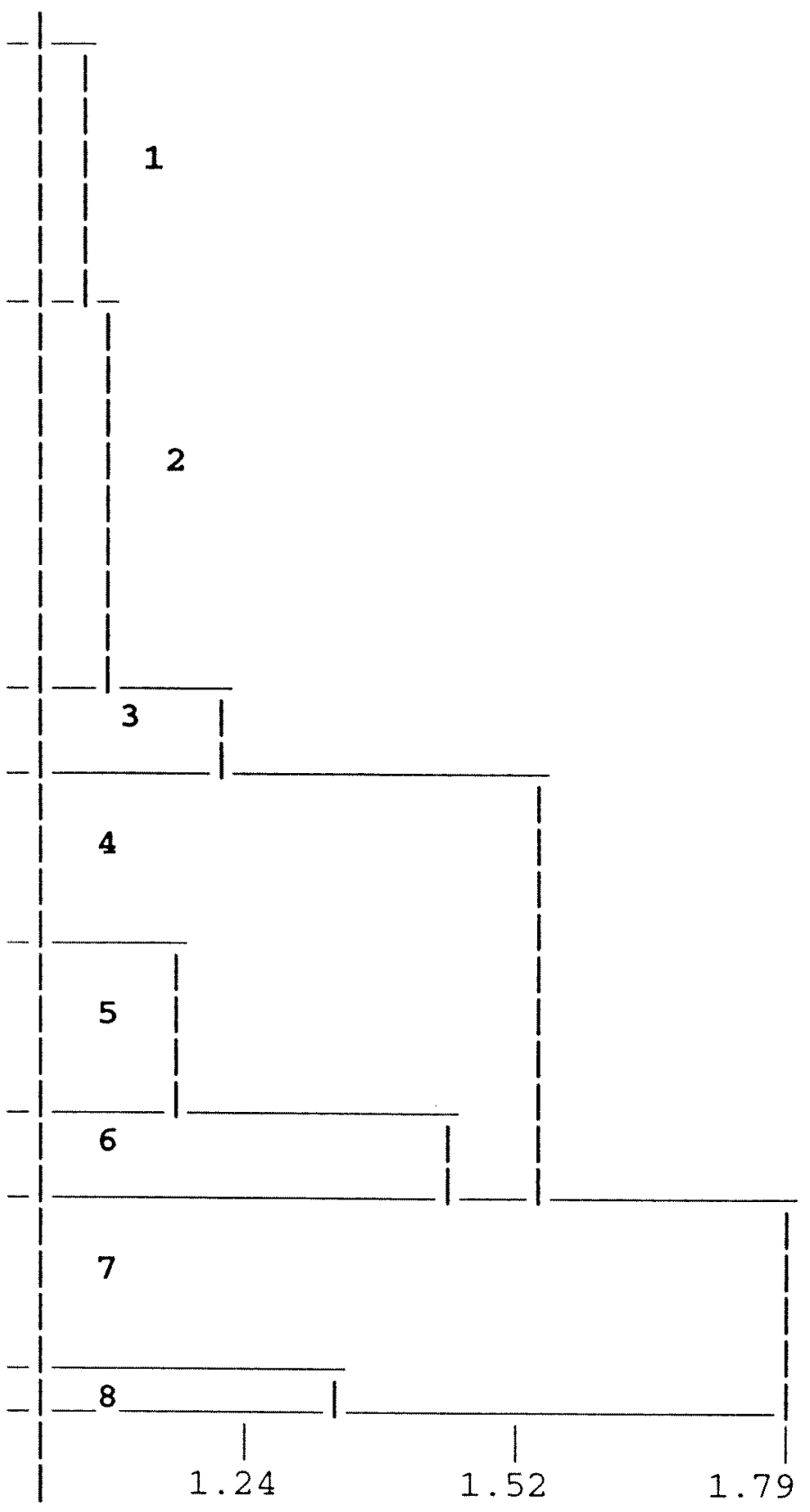

GP05

Dis-similarity Coefficient

Figure 8 Dendrogram derived by classifying 240 quadrats according to their species composition. Structure to the 8 partition level is shown. Matrix was presence/absence data for 400 species and excluded singleton species, fresh-water swamps $(L F=1)$, severely salt-affected quadrats ( $S A L=3$ and 4 ) other than natural saltflats ( $L F=$ 2), and the three quadrats that were inundated for long periods during the trap program (P104, UN13 and WK02).

axis. As pointed out in Results, these two climatic attributes in combination yield a climatic topography and axis only slightly different from warmest period maximum temperature. However, the climatic components of this pattern could also be explained in terms of broadscale landscape relationships. As pointed out in Methods, the ES and part of the GP survey areas are not actually on the Yilgarn Craton; nor are $\mathrm{NO}$ and $\mathrm{DN}$ in the north. Furthermore, Mulcahy and Hingston (1961) divided the Craton in the central part of the study area into eastern and western zones because the
Tertiary Plateau is much more dissected in the west due to their different paleoclimatic histories. Also, the western zone has a rejuvenated drainage system that flows westwards, while the eastern zone's ancient drainage system comprises chains of salt lakes and follows Tertiary paleodrainage lines that are orientated northwards and/or eastwards (e.g. Beard, 2000). These broad landscape differences more or less correspond to the dendrogram partitions analysed (Table 2); deductive studies such as this do not eliminate alternative hypotheses (Oksanen, 2001). 
It was clear that the saltflats support an array of specialist halophilic araneomorphs (species group 18 in Appendix 4). This is not surprising because groundwater dating in south-western Australia (Commander et al., 1994) and sedimentary studies on gypsum accumulation in Lake Lefroy by Zheng et al. (1998) suggest that most halite lakes have been saline for up to a million years (and earlier phases of salt lake formation in this old and geologically stable landscape are probable).

This study revealed an inverse relationship (correlation) between araneomorph species richness and soil salinity, both natural salinity and the secondary salinization induced by agricultural clearing during the last 100 years. Given that saltaffected sites in our study contained a compositional sub-set of their unaffected counterparts (McKenzie et al., 2003), the effect of secondary salinity on araneomorph richness (and composition) is essentially negative. While the araneomorphs belonging to the family Lycosidae (wolf spiders) showed a positive correlation, the relationship is probably not causal. Individual lycosid species, both in Australia and elsewhere, are often confined to particular habitats such as riverine margins, sand dunes and salt lakes (McKay, 1979; Hudson and Adams, 1996; Moring and Stewart, 1994). Generally, Main (2001) characterised lycosids as adaptive opportunists that favour open environments, and predicted that the family would increase in lands cleared for agriculture. As with clearing, rising ground-water salinity results in a net loss of vegetation cover and, in the wheatbelt, our data suggest that these conditions favour lycosids, at least initially. Thus, salt affected sites (mainly the woodland sites on the dissection valley floor, landform 3) hold their lycosid diversity and, at least in the initial stages of secondary salinity, may be further enriched through colonisation by saltflat specialist species such as Lycosa salifodina.

The only substrate patterns that emerged clearly from the quadrat analyses were related to salinity attributes. No distinct, study area-wide patterns in composition emerged that related to other substrate attributes (e.g. soil texture, chemical attributes etc). A plausible explanation is that local endemism, as noted in the salticid and zodariid components (Durrant, 2004; Guthrie and Waldock, 2004), perhaps exaggerated by local covariance in population densities etc, masked any such subtleties.

Broader relationships with soil-landscape zones and climatic gradients were discussed earlier. In any case, clear-cut patterns should not be expected because a species' presence or absence at a site is actually determined by physiological limits and availability of suitable resources in combination with guild, predation and other ecological processes. Rosensweig (1992) pointed out that species define habitats, not vice versa. Correlations with environmental parameters such as those revealed in this study, are indirect consequences of species niche requirements rather than directly causal to their presence/absence at sites.

\section{ACKNOWLEDGEMENTS}

We thank J.K. Rolfe, W.P. Muir, P. van Heurck and $\mathrm{N}$. Hall for assistance in installation and operation of the pit traps; L. King, E. Ladhams and $P$. van Heurck for assistance with sorting, data compilation and field sampling; J.K. Rolfe for data base development and interrogation, B. Baehr for assistance in the identification of some Zodariidae; and M.R. Williams and B. Brand for statistical advice. We thank two anonymous referees for their comments on the manuscript. Funding for this study was provided as part of the State Salinity Strategy.

\section{REFERENCES}

Austin, M.P. (1984). Problems of vegetation analysis for nature conservation. In Myers, K. and Margules, C.R. (eds), Survey methods for nature conservation, vol. 1: 101-130. CSIRO Division of Water and Land Resources, Canberra.

Austin, M.P. (1991). Vegetation theory in relation to costefficient survey. In Margules, C.R. and Austin, M.P. (eds), Nature conservation: cost effective biological surveys and data analysis: 17-22. CSIRO Division of Wildlife and Ecology, Canberra.

Austin, M.P. and McKenzie, N.L. (1988). Data analysis. In Gunn, R.H., Beattie, J.A., Reid, R.E. and van der Graaff, R.H.M. (eds), Australian soil and land survey handbook: guidelines for conducting surveys: 210-232. Inkata Press, Melbourne.

Baehr, B. (2003a). Revision of the tropical genus Tropasteron gen. nov. of north Queensland (Araneae, Zodariidae): a new genus of the Asteron-complex. Memoirs of the Queensland Museum 49: 29-64.

Baehr, B. (2003b). Three new endemic genera of the Asteron-complex (Araneae: Zodariidae) from Australia: Basasteron, Euasteron and Spinasteron. Memoirs of the Queensland Museum 49(1): 1-27.

Baehr, B. (in press). Revision of the new Australian genus Holasteron (Araneae, Zodariidae): taxonomy, phylogeny and biogeography. Memoirs of the Queensland Museum.

Baehr, B. and Baehr, M. (1987). The Australian Hersiliidae (Arachnida: Araneae): taxonomy, phylogeny, zoogeography. Invertebrate Taxonomy 1 : $351-437$.

Baehr, B. and Baehr, M. (1989). Three new species of genus Tamopsis Baehr \& Baehr from Western Australia (Arachnida, Araneae, Hersiliidae). Second supplement to the revision of the Australian Hersiliidae. Records of the Western Australian Museum 14: 309-320.

Baehr, B. and Baehr, M. (1992). New species and new 
records of genus Tamapsis Bachr \& Bachr (Arachnida, Araneace, Hersilidae). Third supplement to the revision of the Australian Hersiliidac. Records of the Western Australian Musem 16: 61-77.

Bachr, B. and Baehr, M. (1993). New species and new records of Hersiliidae from Australia, with an updated key to all Australian species (Arachnida: Arancae: Hersiliidae). Fourth supplement to the revision of the Australian Hersiliidae. Records of the Western Australian Musenm 16: 347-391.

Baehr, B. and Bachr, M. (1995). New species and new records of Hersiliidae from Australia, with an updated key to all Australian species (Arachnida: Araneae: Hersilidae). Fifth supplement to the revision of the Australian Hersiliidae. Records of the Western Australian Museum. Supplement 52: 107-118.

Bachr, B. and Baehr, M. (1998). New species and new records of Hersiliidae from Australia, with an updated key to all Australian species (Arachnida: Araneae: Hersilidae). Sixth supplement to the revision of the Australian Hersiliidae. Records of the Western Australian Muscum 19: 13-38.

Baehr, B. and Churchill, T.B. (2003). Revision of the endemic Australian genus Spinasteron (Araneae, Zodariidae): taxonomy, phylogeny and biogeography. Meretelerate Systematics 17: 641-665.

Baehr, B. and Jocqué, R. (2000). Revisions of genera in the Asteron-complex (Araneae: Zodariidae). The new genera Carasteron and Minasteron. Records of the Western Australian Museum 20: 1-30.

Baehr, B. and Jocqué, R. (2001). Revisions of genera in the Asteron-complex (Araneae: Zodariidae): new genera Pentasteron, Phenasteron, Leptasteron and Subasteron. Memoirs of the Queensland Musetum 46: 359-385.

Baxter, J.L. and Lipple, S.L. (1985). Perenjori, Western Australia 1:250 000 geological series. Geological Survey of Western Australia, Perth.

Beard, J.S. (1980). A new phytogeographic map for Western Australia. Western Australian Herbarium Research Notes 3: 37-58.

Beard, J.S. (1981). Vegetation surity of Western Australia Swan 1 : 1000 000 vegetation series. University of Western Australian Press, Nedlands.

Beard, J.S. (1990). Plant lifi of Western Australia. Kangaroo Press, Kenthurst.

Beard, J.S. (2000). Drainage evolution in the Moore Monger system, Western Australia. Joumal of the Royal Society of Western Australia 83: 29-38.

Belbin, L. (1995). PATN technical reference. CSIRO, Canberra.

Brennan, K.E.C. (1999). Discovery of the spider Ambicodamus marac (Araneae: Nicodamidae) in the northern jarrah forest of Western Australia. Records of the Western Australian Museum 19: 323-325.

Brennan, K.E.C. and Majer, J.D. (in press). Conservation and biodiversity of spiders in Western Australian jarrah forest: untangling multiple disturbance effects from burning and mining. In Lunney, D. (ed.), Consertation of Australia's Fore'st Fama, $2^{\text {ma }}$ edn. Roval Zoological Society of New South Wales, Sydney.

Brennan, K.E.C., Majer, J.D. and Moir, M.L. (in press).
Biodiversity of jarrah forest spiders at Jarrahdale, Western Australia: richness, endemicity and taxonomic knowledge. Pacific Consereation Biohery.

Bureau of Meteorology (2001). What is the weather usually like? Climate averages for Western Australian sites. http://www bom.gov.au/climate/averages/ tables/ca_wa_names.shtml.

Chin, R.J. (1986). Kellerberm, Western Australia, 1:250) o00 geological series. Geological Survey of Western Australia, Perth.

Clarke, K.R. (1993). Non-parametric multivariate analyses of changes in community structure. Australian foumal of Ecology 6: 163-174.

Commander, D.P., Fifield, L.K., Thorpe, P'M., Davic, R.F., Bird, J.R. and Turner, J.V. (1994). Chlorine-36 and carbon-14 measurements on hypersaline groundwater in Tertiary paleochannels near Kalgoorlie, Western Australia. Professional Papers 37: 53-60. Geological Survey of Western Australia, Perth.

Commander, D.P., Schoknecht, N., Verboom, W. and Caccetta, P. (2002). The geology, physiology and soils of wheatbelt valleys. $I n$ V. Read \& Associates (eds), Dealing with salinity in wheatbolt ialleys. Processes, prospects and practical options: 1-21. Waters and Rivers Commission, Perth (Available on CD).

Crekanowski, J. (1932). Coefficient of racial likeness, und durchschnittliche differenz. Anthropologischer Anzeiger 9: $227-249$.

Davies, V.T. (1994). The huntsman spiders Heteropoda Latreille and Yiinthi gen. nov. (Araneae: Heteropodidae) in Australia. Memoirs of the Quensland Muscum 35: 75-122.

Davies, V.T. and Żabka, M. (1989). Illustrated keys to the genera of jumping spiders (Araneae: Salticidae) in Australia. Memoirs of the Que'nsland Muse'um 27: 189 266.

Durrant, B.J. (2004). Biogeographical patterns of zodariid spiders (Araneae: Zodariidae) in the wheatbelt region, Western Australia. Records of the Western Australian Museum Supplement 67:217-230

Durrant, B.J. and Guthrie, N.A. (2004). Faunas of unflooded saline wetland floors of the Western Australian wheatbelt. Records of the Western Australian Muserum Supplement 67: 231-256.

Environment Australia (2000). Reriston of the interim biogergraphic regionalisation for Australia (IBRA) and declopment of ersion 5.1. Environment Australia, Canberra.

Faith, D.P., Minchin, P.R. and Belbin, L. (1987). Compostional dissimilarity as a robust measure of ecological distance: a theoretical model and computer simulations. Vesetatio 69: 57-68.

Forster, R.R. (1959). The spiders of the family Symphytognathidae. Transactions of the Royal Seriety of Nere Zivland 86: 269-329.

Forster, R.R., Millidge, A.F. and Court, D.J. (1988). The spiders of New Zealand. Part VI. Otage Musem Bulletin 6: 1-124.

Forster, R.R. and Platnick, N.I. (1985). A review of the austral spider family Orsolobidae (Arachnida, Araneae), with notes on the superfamily Dysderoidea. Bulletin of the American Musem of Natural History 181: 1-230. 
Framenau, V.W. (2002). Revision of the wolf spider genus Artoria Thorell (Araneae: Lycosidae). Ine'rtebrate Systematics 16: 209-235.

Framenau, V.W. and Vink, C.J. (2001). Revision of the wolf spider genus Venatrix Roewer (Araneae: Lycosidae). Inertebrate Taxonomy 15: 927-970

George, R. and Coleman, M. (2002). Hidden menace or opportunity - groundwater hydrology, playas and commercial options for salinity in wheatbelt valleys. In V. Read \& Associates (eds), Denling with salinity in wheatbelt walleys. Processes, prospects and practical options: 1-21. Waters and Rivers Commission, Perth (Available on CD).

George, R.J., McFarlane, D.J. and Speed, R.J. (1995). The consequences of a changing hydrologic environment for native vegetation in Western Australia. In Saunders, D.S., Craig, J.L. and Mattiske, E.M. (eds), The role of networks: 9-22. Surrey Beatty \& Sons: Chipping Norton, NSW

Gray, M.R. (1994). A review of the filistatid spiders (Araneae: Filistatidae) of Australia. Records of the Australian Museun 46: 39-61.

Griswold, C.E. (2001). A monograph of the living world genera and Afrotropical species of cyatholipid spiders (Araneae, Orbiculariae, Araneoidea, Cyatholipidae). Menoirs of the California Academy of Sciences 26: 1-251.

Guthrie, N.A. and Waldock, J.M. (2004). Patterns in the composition of the jumping spider (Arachnida: Araneae: Salticidae) assemblage from the Wheatbelt region, Western Australia. Records of the Western Australian Museum, Supplement 67: 203-216.

Harvey, M.S. (1995). The systematics of the spider family Nicodamidae (Araneae: Amaurobioidea). Invertebrate Taxonomy 9: 279-386.

Harvey, M.S. (2002). Short-range endemism in the Australian fauna: some examples from non-marine environments. Invertebrate Systematics 16: 555-570.

Harvey, M.S., Sampey, A., West, P.L.J. and Waldock, J.M. (2000). Araneomorph spiders from the southern Carnarvon Basin, Western Australia: a consideration of regional biogeographic relationships. Records of the Western Australian Museum Supplenent 61: 295-321.

Hatton, T.J. and Ruprecht, J. (2001). Watching the rivers flow: hydrology of the wheatbelt. In V. Read \& Associates (eds), Dealing with salinity in wheatbelt aalleys. Processes, prospects and practical options: 1-15. Waters and Rivers Commission, Perth (Available on CD).

Hickman, V.V. (1944). On some new Australian Apneumomorphae with notes on their respiratory systems. Papers and Procedings of the Royal Society of Tasmania 1943: 179-195.

Hickman, V.V. (1945). A new group of apneumone spiders. Transactions of the Connecticut Academly of Arts and Scionces 36: 135-157.

Hickman, V.V. (1979). Some Tasmanian spiders of the families Oonopidae, Anapidae and Mysmenidae. Papers and Procedings of the Royal Society of Tasmania 113: $53-79$.

Hirst, D.B. (1989). A revision of the genus Pedima Simon (Heteropodidae: Araneae) in Australia. Recorts of the South Australian Museum 23: 113-126.
Hirst, D.B. (1991a). Revision of Australian species of the genus Holconia Thorell (Heteropodidae: Araneae). Records of the South Australian Museum 24: 91-109.

Hirst, D.B. (1991b). Revision of the Australian genera Eotelena Hogg and Znchria L. Koch (Heteropodidae: Araneae). Records of the South Australian Museum 24: 91-109.

Hirst, D.B. (1992). Revision of the genus Isopeda Koch (Heteropodidae: Araneae) in Australia. Inetertebrate Taxonomy 6: 337-387.

Hopper, S.D., Harvey, M.S., Chappill, J.A., Main, A.R. and Main, B.Y. (1996). The Western Australian biota as Gondwanan heritage - a review. In Hopper, S.D., Chappill, J.A, Harvey, M.S. and George, A.S. (eds), Gondwann heritage: past, present and futtre of the Westem Australian biota: 1-46. Surrey Beatty \& Sons, Sydney.

Hudson, P. and Adams, M. (1996). Allozyme characteristics of the salt lake spiders ( $L y \cos a$ : Lycosidae: Araneae) of southern Australia: systematic and population genetic implications. Australian Journal of Zoology 44: 535-567.

Jocqué, R. (1995a). Notes on Australian Zodariidae (Araneae), I. New taxa and key to the genera. Records of the Australian Muse'um 47: 117-140.

Jocqué, R. (1995b). Notes on Australian Zodariidae (Araneae), II. Redescriptions and new records. Records of the Australian Muscum 47: 141-160.

Jocqué, R. and Baehr, B. (2001). Revisions of genera in the Asteron-complex (Araneae: Zodariidae). Asteron Jocqué and the new genus Pseudasteron. Records of the Australian Museum 53: 21-36.

Jocqué, R. and Baehr, M. (1992). A revision of the Australian spider genus Storena (Araneae: Zodariidae). Inzertebrate Taxonomy 6: 953-1004.

Main, B.Y. (1991). Kimberley spiders: rainforest strongholds. In McKenzie, N.L., Johnston, R.B. and Kendrick, P.G. (eds), Kimberley rainforests: 271-293. Surrey Beatty \& Sons, Sydney.

Main, B.Y. (2001). Historical ecology, responses to current ecological changes and conservation of Australian spiders. Journal of Insect Conservation 5: 9-25.

Main, B.Y., Sampey, A. and West, P.L.J. (2000). Mygalomorph spiders of the southern Carnarvon Basin, Western Australia. Records of the Western Australian Museum Supplement 61: 281-293.

McKay, R.J. (1979). The wolf spiders of Australia (Araneae: Lycosidae): 12. Descriptions of some Western Australian species. Memoirs of the Quensland Museum 19: 241-275.

McKenzie, N.L., Burbidge, A.H. and Rolfe, J.K. (2003). Effect of salinity on small, ground-dwelling animals in the Western Australian wheatbelt. Australian Joumal of Botany 51: 725-740.

McKenzie, N.L., Gibson, N., Keighery, G.J. and Rolfe, J.K. (2004). Patterns in the biodiversity of terrestrial environments in the Western Australian wheatbelt. Records of the Western Australian Museum Supplement 67: 293-335.

McKenzie, N.L., Robinson, A.C. and Belbin, D.L. (1991). Biogeographic survey of the Nullarbor district, Australia. In Margules, C.R. and Austin, M.P. (eds), 
Nature Conserention: Cost Effective Biolosical Surreys and Data Analysis: 109-126. CSIRO Division of Wildlife and Ecology: Canberra.

McMahon, J.P., Hutchinson, M.F., Nix, H.A. and Ord, K.D. (1995). ANUCLIM users guide, version 1. Centre for Resource and Environmental Studies, Australian National University: Canberra.

Morgan, G. (2001). Landscape health in Australia. A rapid assessment of the relative condition of Australia's bioregions and subregions. Environment Australia and National Land and Water Resources Audit, Canberra.

Morgan, K.H. and Peers, R. (1973). Esperance-Mondrain Island, Western Australia, 1:250 000 geological series. Geological Survey of Western Australia, Perth.

Moring, J.B. and Stewart, K.W. (1994). Habitat partitioning by the wolf spider (Araneae, Lycosidae) guilds in streamside and riparian vegetation zones of the Conejos River, Colorado. Journal of Arachnology 22: $205-217$.

Mulcahy, M.J. and Hingston, F.J. (1961). The development and distribution of the soils of the YorkQuairading area, Western Australia, in relation to landscape evolution. Soil Publication 17. CSIRO, Canberra, Australia.

Myers, J.S. and Hocking, R.M. (1998). Geological Map of Western Australia $1: 2,500,000$ (13 $3^{\text {th }}$ edn). Geological Survey of Western Australia, Perth.

Myers, N., Mittermeier, R.A., Mittermeier, C.G., Fonseca, G.A.B. da and Kent, J. (2000). Biodiversity hotspots for conservation priorities. Nature 403: 853-858.

Newsome, A.E. and Catling, P.C. (1979). Habitat preference of mammals inhabiting heathlands of warm temperate coastal, montane and alpine regions of southeastern Australia. In Specht, R.L. (ed.), Ecosystems of the world, vol. $9 \mathrm{~A}$. Heathlands and related shrublands of the world: 301-316. Elsevier, Amsterdam.

Oksanen, L. (2001). Logic of experiments in ecology: is pseudoreplication a pseudoissue? Oikos 94: 27-38

Platnick, N.I. (2000). A relimitation and revision of the Australasian ground spider family Lamponidae (Araneae: Gnaphosoidea). Bulletin of the American Museum of Natural History 254: 1-330.

Platnick, N.I. (2002). A revision of the Australasian ground spiders of the families Ammoxenidae, Cithaeronidae, Gallieniellidae and Trochanteriidae (Araneae: Gnaphosoidea). Bulletin of the American Museum of Natural History 271: 1-243.

Platnick, N.I. (2004). The World Spider Catalog, Version 4.5, American Museum of Natural History, New York at http://research.amnh.org/entomology/spiders/ catalog/index.html. http//research.amnh.org/ entomology/spiders/catalog81-87/index.html

Platnick, N.I. and Forster, R.R. (1989). A revision of the temperate South American and Australasian spiders of the family Anapidae (Araneae, Araneoidea). Bulletin of the American Museum of Natural History 190: $1-139$.
Raven, R.J., Baehr, B.C. and Harvey, M.S. (2002). Spiders of Australia: interactive identification to subfamily. CSIRO/ABRS, Melbourne.

Rosensweig, M.L. (1992). Species diversity gradients: we know more and less than we thought. Journal of Mammalogy 73: 715-730.

Santos, A.J. and Gonzaga, M.O. (2003). On the spider genus Oecobius Lucas, 1846 in South America (Araneae, Oecobiidae). Journal of Natural History 37: 239-252.

Schoknecht, N. (2002). Soil groups of Western Australia, edn 3. Resource Management Technical Report 246. Department of Agriculture, Perth.

Simon, E. (1908). Araneae, $1^{\text {ro }}$ partie. In Michaelsen, W. and Hartmeyer, R. (eds), Die Fama SüdwestAustraliens, vol. 1: 359-446. Gustav Fischer, Jena.

Simon, E. (1909). Araneae, $2^{\text {me }}$ partie. In Michaelsen, W. and Hartmeyer, R. (eds), Die Fauna SüdwestAustraliens, vol. 2: 155-212. Gustav Fischer, Jena.

Sneath, P.H.A. and Sokal, R.R. (1973). Numerical taxonomy: the principles and practice of numerical classification. Freeman, San Francisco.

Statsoft (2001). STATISTICA System Reference. Statsoft, Tulsa, USA.

Vink, C.J., Mitchell, A.D. and Paterson, A.M. (2002). A molecular analysis of phylogenetic relationships of Australasian wolf spider genera (Araneae: Lycosidae). Joumal of Arachnology 30: 227-237.

Żabka, M. (1990). Salticidae (Arachnida: Araneae) of Oriental, Australian and Pacific regions, IV. Genus Ocrisiona Simon, 1901. Records of the Australian Museum 42: 27-43.

Żabka, M. (1991). Salticidae (Arachnida: Araneae) of Oriental, Australian and Pacific regions, V. Genus Holoplatys Simon, 1885. Records of the Australian Museum 43: 171-240.

Żabka, M. (1992a). Salticidae (Arachnida: Araneae) of Oriental, Australian and Pacific regions, VIII. A new genus from Australia. Records of the Western Australian Museum 15: 673-684.

Żabka, M. (1992b). Salticidae (Arachnida: Araneae) of Oriental, Australian and Pacific regions, VII. Paraplatoides and Grayenulla - new genera from Australia and New Caledonia. Records of the Australian Museum 44: 165-183.

Zabka, M. (2000). Salticidae (Arachnida: Araneae) of the Oriental, Australian and Pacific regions, XIII: the genus Sandalodes Keyserling. Invertebrate Taxonomy 14: 695-704.

Żabka, M. (2001). Salticidae (Arachnida: Araneae) of Oriental, Australian and Pacific regions, XIV. The genus Adoxotoma Simon. Records of the Western Australian Museum 20: 323-332.

Zheng, H.B., Wyrwoll, K.H., Li, C.M. and Powell, C.M. (1998). Onset of aridity in southern Western Australia - a preliminary palaeomagnetic appraisal. Global and Planatery Change 18: 175--187.

\section{Electronic appendices are on $C D$ inside the back cover}

